\title{
Brg1 controls neurosensory cell fate commitment and differentiation in the mammalian
}

\section{inner ear}

Jinshu Xu ${ }^{1, \#}$, Jun $\mathrm{Li}^{1, \#}$, Yong-Hwee Eddie Loh $^{2}$, Ting Zhang ${ }^{1}$, Huihui Jiang ${ }^{1}$, Bernd Fritzsch ${ }^{3}$, Aarthi Ramakrishnan ${ }^{2}$, Li Shen ${ }^{2}$ and Pin-Xian $\mathrm{Xu}^{1,4, *}$

${ }^{1}$ Department of Genetics and Genomic Sciences, ${ }^{2}$ Department of Neurosciences and

${ }^{4}$ Developmental and Regenerative Biology, Icahn School of Medicine at Mount Sinai, New York, NY 10029, USA

${ }^{3}$ Department of Biology, Center on Aging \& Aging Mind and Brain Initiative, The University of

Iowa, Iowa City, Iowa 52242-1324

${ }^{\#}$ Authors contributed equally to this work.

*Author for correspondence:

Pin-Xian Xu, Ph.D.

Department of Genetics and Genomic Sciences

Icahn School of Medicine at Mount Sinai

East Building Room 14-20D

1425 Madison Ave

New York, NY10029

Tel: $\quad 212-659-6787$

Fax: 212-849-2508

e-mail: pinxian.xu@mssm.edu 


\begin{abstract}
Otic ectoderm gives rise to almost all cell types of the inner ear; however, the mechanisms that link transcription factors, chromatin, lineage commitment and differentiation capacity are largely unknown. Here we show that Brg1 chromatin-remodeling factor is required for specifying neurosensory lineage in the otocyst and for inducing hair and supporting cell fates in the cochlear sensory epithelium. Brg1 interacts with the critical neurosensory-specific transcription factors Eya1/Six1, both of which simultaneously interact with BAF60a or BAF60c. Chromatin immunoprecipitation-sequencing (ChIP-seq) and ChIP assays demonstrate Brg1 association with discrete regulatory elements at the Eyal and Sixl loci. Brgl-deficiency leads to markedly decreased Brg1 binding at these elements and loss of Eyal and Sixl expression. Furthermore, ChIP-seq reveals Brg1-bound promoter-proximal and distal regions near genes essential for inner ear morphogenesis and cochlear sensory epithelium development. These findings uncover essential functions for chromatin-remodeling in the activation of neurosensory fates during inner ear development.
\end{abstract}

\title{
Introduction
}

The mammalian inner ear uses sensory hair cells (HCs) for mechanotransduction of vestibular and auditory stimuli and transmits this information to the brain via sensory neurons. Precursor cells for sensory cells and neurons are specified in the epithelium of the otocyst, which develops from the otic placode - thickened ectoderm that forms adjacent to the hindbrain at $\sim \mathrm{E} 8.5$ and differentiates to form all inner ear structures. Neurosensory domain within the ventral region of the otic ectoderm is defined by transcription factors (TFs) Eya1 and Six1. A subpopulation of their daughter cells is induced to become neuroblasts from $\sim \mathrm{E} 9.0-9.25$, which is marked by the 
basic helix-loop-helix (bHLH) TF Neurog1. These progenitors proliferate and differentiate to become Neurod $1^{+}$and then delaminate into the mesenchyme to aggregate and form the spiral and vestibular ganglion. In contrast, the prosensory progenitors continue to divide and expand within a common prosensory primordium until the commitment of sensory $\mathrm{HC}$ fate, which is marked by the bHLH protein Atoh1 from E12.5 in the vestibule and E14.5 in the cochlea. Sox2 is a crucial TF necessary for prosensory progenitor specification (Kiernan et al., 2005). We have shown that the transcription coactivator Eya1 and the homeodomain protein Six1 form a key transcriptional complex as neuronal or sensory HC determinants by demonstrating that expression of these two proteins can convert cochlear nonsensory epithelial GER (greater epithelial ridge) cells to a hair or neuronal cell fate through interaction with different partner proteins. Eya1-Six1 interacts with Sox2 to cooperatively activate an Atoh1-regulatory network for HC fate induction (Ahmed et al., 2012a) or with Brg1-BAFs (Brg1/Brm-associated factors) chromatin-remodeling proteins of the SWI/SNF (Switch/Sucrose non-fermentable) family to activate a Neurog1-Neurod1 regulatory network for neuronal differentiation (Ahmed et al., 2012b). Mutations in these factors cause congenital sensorineural hearing loss in humans or deafness in mice (Abdelhak et al., 1997; Hagstrom et al., 2005; Ozaki et al., 2004; Ruf et al., 2004; Xu et al., 1999; Zheng et al., 2003). However, the mechanisms that control the expression of Eya1, Six 1 and Sox 2 during acquisition of distinct cell fates are not understood. Furthermore, the SWI/SNF family plays a vital role in facilitating binding of specific TFs to nucleosomal DNA, yet no studies have been performed to directly address its roles in neurosensory cell fate specification and differentiation during inner ear development.

The SWI/SNF complex consists of multiple general and facultative subunits, whose activity requires Brg1 or the related Brm as ATPase (Ho and Crabtree, 2010). While Brm-null 
mice are viable and normal (Muchardt et al., 1998), deletion of Brgl causes early periimplantation lethality (Bultman et al., 2000). Brg1 has been linked to progenitor cell proliferation, differentiation and survival in a variety of organs, including the heart, central nervous system, muscle, blood cells, and immune system (see reviews (Hargreaves and Crabtree, 2011; Ho and Crabtree, 2010). Since Eya1-Six1 interacts with the Brg1-BAFs, we conditionally deleted the central catalytic subunit Brg1 in Eyal-expressing cells at different developmental stages to investigate the function of the SWI/SNF complex during inner ear morphogenesis. Here we show that Brg1 is necessary for specifying both neuronal and sensory fates and is required for the expression of Eyal and Six1, both of which act upstream of Sox2. In the developing cochlea, $\operatorname{Brg} 1$ is not only necessary for prosensory domain specification but also for precursor cell differentiation into either HCs or supporting cells (SCs). Co-immunoprecipitation (coIP) shows a selective physical association of Eya1 and Six1 with the variants a and c but not b of the BAF60 structural subunit in the cochlea. Chromatin immunoprecipitation followed by deep sequencing (ChIP-seq) on E13.5 cochleae reveals Brg1-enriched regions both proximal to and distal from the promoters for genes essential for cochlea morphogenesis including Eya1, Six 1, Sox 2, p2 $7^{\text {Kip } 1}$ and Gata3 loci. ChIP assays confirm co-occupancy of BAF60a/c at the Brg1-enriched regulatory elements at these loci. Conditional deletion of Brg1 results in significantly reduced Brg1 binding to these elements and loss of expression of these genes in the inner ear. Together, these results demonstrate that Brg1-based BAF complexes coordinate multiple processes of neurosensory lineage activation and differentiation and provide insight into how Brg1-BAFs act to promote lineage-specific properties in the inner ear.

\section{Results}




\section{Brg1 controls specification of neurosensory progenitors in the otocyst}

Brg1 is widely expressed in the otic placode and otocyst and in the delaminating neurons at E8.5-10.5 (Figure 1A,B). In the developing cochlea, Brg1 is highly expressed in the prosensory primordium marked by Sox2 (Figure 1C) and in differentiating HCs and SCs within the organ of Corti at P0 (Figure 1D). Strong Brg1 expression was also observed in LER (lesser epithelial ridge) and SGNs (spiral ganglion neurons) (Figures 1C,D and Figure 1-figure supplement 1A). To study its role in neurosensory cell development during inner ear morphogenesis, $B r g 1^{f l f l}$ mice (Sumi-Ichinose et al., 1997) were bred with Eyal ${ }^{\text {CreERT2 }}$ to generate Eyal ${ }^{\text {CreERT2 }} ;$ Brg $^{\text {flffl }}$ $\left(B r g 1^{C k o / C k o}\right.$ or CKO) mice with specific deletion of Brg1 in Eya $1^{+}$cells. Anti-Brg1 staining confirmed selective ablation in the ventral otocyst where Eyal is normally expressed (Figure 1figure supplement $1 \mathrm{~B}-\mathrm{E}) . \mathrm{Brg}^{\mathrm{Cko} / \mathrm{Cko}}$ mice displayed a considerably smaller otocyst $(n=6)$ than in their wild-type littermates and an absence of the VII-VIIIth ganglionic complex (Figure 1E). Examination of inner ears at E15.5 showed a degenerated cavity-like structure without neurosensory cells in $\mathrm{Brgl}^{\mathrm{Cko} / \mathrm{Cko}}(\mathrm{n}=6$, Figure $1 \mathrm{~F})$. These data indicate that Brg1 has an essential role in inner ear neurosensory structure formation.

Analysis of EdU (5-ethynyl-2'-deoxyuridine) incorporation (chased for 2 hours before harvest) revealed a reduction in proliferation rate in the mutant to $\sim 57 \%$ of that in wild-type littermates $(37.9 \pm 0.4 \%$ vs $66.1 \pm 0.3 \%$ per otocyst; $n=3$, Figure $1 \mathrm{G}, \mathrm{H})$. TUNEL assay showed increased abnormal cell apoptosis in the mutant $(n=3$, Figure $1 \mathrm{I}, \mathrm{J})$. Thus, the growth arrest and degeneration of the otocyst associated with Brg1-deficiency is due to defective cell proliferation and abnormal apoptosis.

To understand the molecular basis for the otic defects, we performed in situ hybridization (ISH) to characterize genes crucial for neurosensory cell fate specification. Eya1, Six 1 and Sox 2 
are expressed in the ventral region of the otocyst from which all sensory organs develop (Figure 2A-E), whereas the expression of all three genes was not observed in the CKO littermates $(n=6)$. Dlx5, which is expressed in the dorsolateral region of the otocyst ( $n=6$, Figure $2 \mathrm{~F}$ ), was expressed in the ventral region of the CKO otocyst. This indicates that in the absence of Eya1Six1-Sox2-promoted cell fate, the otic epithelium is filled with $D l x 5$-positive cells, suggesting acquisition of a non-sensory fate or expansion of the non-sensory area.

To determine if lack of VIIIth cochleovestibular ganglion formation is due to lack of neuroblast specification or differentiation, we examined the expression of Neurogl and Neurodl by ISH. At $\sim$ E9.25, Neurogl expression in the anterior region of the otocyst was undetectable in $\mathrm{Brgl}^{\mathrm{Cko} / \mathrm{Cko}}$ littermates (Figure $2 \mathrm{G}$ ), but its expression in the Vth ganglion was preserved in the CKO. Neurodl expression, which is detected in all cranial ganglia at E10.5 (Figure 2H), was also lost in the VIIIth ganglion of the CKO $(n=5)$. These data suggest that neuroblasts are not specified in the absence of Brg1. In addition, the IXth and Xth ganglia were not formed, while the VIIth and Vth ganglia were reduced in size in the mutant (Figure 2H). Since this phenotype is similar to that seen in Eyal ${ }^{-/} ;$Sixl $1^{-/-}$double mutant (Zou et al., 2004), altered Neurogl and Neurodl expression could be due to loss of both Eyal and Sixl expression in Brgl ${ }^{\text {Cko/Cko }}$. Together, these data suggest that Brg1 activity is required for the expression of Eyal and Sixl to induce otic ectodermal progenitors to adopt a sensory or neuronal fate.

\section{Sox2 expression depends on both Eya1/Six1 function}

We have previously reported that Sox 2 expression is detectable in the otocyst of Eyal $1^{-/}$or Six $1^{-/-}$ single null embryos (Zheng et al., 2003; Zou et al., 2008). Since otic neuroblast specification requires both Eya1/Six1 function, we hypothesized that Eya1/Six1 may also interact to induce 
Sox 2 activation in the otic placode to promote a sensory fate. Indeed, Sox2 expression was not detected in Eyal $^{-/-} ;$Sixl $^{-/}$double mutant otic placode and otic cup between E8.5-9.25 (Figure 2I,J). However, Dlx5 expression was similarly detected in the entire ectodermal region (Figure $2 \mathrm{~K}, \mathrm{~L}$ ), differing from its restricted dorsal expression in wild-type littermates (Figure 2L). In contrast, conditional deletion of Sox 2 using Eyal ${ }^{\text {CreERT2 }}$ did not cause a ventral shift of Dlx5 expression in the otocyst (Figure 2-figure supplement 2A-D). Both Eyal and Six 1 expression were observed in the ventral region of Sox $2^{C k o / C k o}$ otocyst (Figure 2-figure supplement 2E-H), confirming that Eya1/Sixl function upstream of Sox2. Thus, the significant reduction of Eyal/Sixl expression in the Brgl CKO may in turn cause loss of Sox 2 expression. Together, these data indicate that Eya1/Six 1 interact synergistically to induce Sox 2 expression in early otic progenitors to support the Eya1/Six1/Brg1 mediated prosensory fate specification.

\section{Brg1 specifies the prosensory primordium in the cochlear epithelium}

We next investigated if Brg1 has a role in auditory prosensory primordum establishment during cochlear morphogenesis. The cochlear duct elongates from the ventral region of the otocyst and the prosensory progenitors proliferate until reaching a defined number. They then exit the cell cycle to become $\mathrm{p} 27^{\mathrm{Kip} 1+}$ precursors to form a non-proliferating precursor domain in an apex to base progression between E12.5-E14.5 (Chen et al., 2002). This domain appears as a three- to four-cell layered epithelium marked by Sox2/p27 ${ }^{\text {Kip1 }}$ (Figure 3A,E). In E14.5 Brg1 CKO given tamoxifen (Tam) at E11.75-12.5, Brg1 was selective ablation in the Eya1-expressing domains including the prosensory domain, GER and SGNs (Figure 1-figure supplement 1F,G). Sox $2^{+}$ progenitors were present along the entire cochlear duct, which was shortened in the CKO (Figure 3B-D). However, only a few Sox $2^{+}$cells in the apex to medial region of the cochlea became 
$\mathrm{p} 27^{\mathrm{Kip} 1+}$ (Figure 3B-D). The prosensory primordium on sections appeared slightly thinner along the basoluminal axis and Sox 2 expression was maintained in the precursors in the basal layer but appeared to be downregulated in the cells in the luminal layer (Figure 3F-H), a process normally associated with $\mathrm{HC}$ differentiation. Within the primordial organ of Corti, only cells in the medial region were positive for $\mathrm{p} 27^{\mathrm{Kip} 1}$ (Figure 3B,C,F-H). As cycle exit of the progenitors occurs in an apex-to-base and medial-to-lateral direction from E12.5, the few $\mathrm{p} 27^{\mathrm{Kip} 1+}$ cells observed in the CKO cochlea could be due to the presence of Brg1 activity in those cells when CreERT2 was induced, as it takes at least $6 \mathrm{hrs}$ for the CreER fusion protein to translocate to the cell nucleus and the peak marking occurs over a subsequent period of 12-24 hrs (Danielian et al., 1998). Consistent with apex to base upregulation of $\mathrm{p} 27^{\mathrm{Kip} 1}$, we found only very few $\mathrm{p} 27^{\mathrm{Kip} 1+}$ cells in the apical tip of the CKO cochlea after Tam injection at $\sim \mathrm{E} 11.75$ (Figure. 3D). Thus, Brg1 is necessary to specify the prosensory primordium by regulating $\mathrm{p} 27^{\mathrm{Kip} 1}$ activation. In the absence of Brg1, Sox $2^{+}$progenitors fail to become $\mathrm{p} 27^{\mathrm{Kip} 1+}$ precursors.

We further investigated whether the arrest of cochlear growth is due to either a decrease in proliferating cells or an increase in apoptosis. EdU incorporation demonstrated a decrease of proliferating Sox $2^{+}$cells in the CKO sensory epithelium at E13.5-14.5 after Tam injection at $\sim$ E11.5 (by $\sim 83 \%$ ) (Figure 3-figure supplement 3A). Quantification of the $\mathrm{TUNEL}^{+}$cells in the epithelium located in the floor of cochlear duct found a strong increase in apoptosis (5.2-fold) (Figure 3-figure supplement 3B). Thus, Brg1 activity is also required for both cell proliferation and cell survival in the cochlear sensory epithelium.

\section{Differentiation of the postmitotic precursors into HCs or SCs is disrupted in $\mathrm{Brg} 1^{\text {Cko/Cko }}$}

We next asked whether the $\mathrm{p} 27^{\mathrm{Kip} 1+}$ or $\mathrm{p} 27^{\mathrm{Kip} 1-}$ precursors in the mutant presumptive organ of 
Corti are competent for $\mathrm{HC}$ or $\mathrm{SC}$ differentiation. Myo7a is a marker specific for differentiating HCs, while Prox 1 is a SC marker specific for pillar cells lying between inner and outer HCs and three Deiters' cells underlying outer HCs (Figure 4-figure supplement 4). Double immunostaining showed neither Myo $7 \mathrm{a}^{+} \mathrm{HCs}$ nor Prox $1^{+} \mathrm{SCs}$ present in $\mathrm{Brgl}^{\mathrm{CKo} / \mathrm{Cko}}$ cochlea at E18.5 (Tam at E11.75-12.5) (Figure 4-figure supplement 4). Sox2 expression had also disappeared in the mutant by this stage (data not shown). Thus, in the absence of Brg1 the prosensory progenitor cells fail to either exit cell cycle or enter terminal differentiation, eventually leading to loss of Sox2 expression.

We next administered Tam more than a day later, from $\sim$ E13.5-14.0, to examine the role of $\mathrm{Brg} 1$ in the activation of $\mathrm{HC}$ and $\mathrm{SC}$ fates during terminal differentiation of the postmitotic precursors, which initiates near the base from $\sim$ E14.5. By E18.0, the cochlea had reached a full 1.5 turns, and differentiation of HCs, which are aligned into one row of inner and three rows of outer HCs, had reached the apical tip as outlined by Myo7a (Figure 4A,B). In Brg1 ${ }^{C k o / C k o}$, while the cochlea was considerably smaller and only reached $\sim 1$ turn, very few HCs that failed to align into the characteristic rows of inner and outer HCs were present in the base, and none were observed in the medial to apex (Figure 4A,B). Since HC differentiation occurs in a medial-tolateral (inner-to-outer) gradient, the Myo $7 \mathrm{a}^{+} \mathrm{HCs}$ likely represent the inner $\mathrm{HCs}$, which begin to differentiate at least more than a day prior to outer HCs. Indeed, immunostaining for calretinin, a marker specific for inner $\mathrm{HCs}$, confirmed that all $\mathrm{Myo} \mathrm{a}^{+} \mathrm{HCs}$ in the base were also calretinin ${ }^{+}$ (Figure 4C). As seen on sections, the organ of Corti was patterned into a two-cell layered epithelium with one-cell layer of HCs on the luminal side and one cell layer of underlying SCs (Figure 4C). In $B r g 1^{C k o / C k o}$, no obvious change in the epithelial thickness along the basoluminal axis was observed. However, cells in the presumptive organ of Corti were irregularly patterned 
and disorganized with morphological alteration in the $\mathrm{CKO}$ compared to those in the control littermates (Figure 4C). Thus, Brg1 is necessary for HC fate induction and sensory epithelium formation in the cochlea.

The SCs maintain high levels of $\mathrm{p} 27^{\mathrm{Kip} 1}$ and Sox 2 through adult stage (Figures 5A,B). Although the SCs in the CKO were irregularly shaped and the organ of Corti became slightly narrower compared to that in wild-type controls, they expressed Sox $2 / \mathrm{p} 27^{\mathrm{Kip} 1}$. Prox 1 was expressed in Brgl CKO SCs in the base but not in the medial to apex, and the Prox $1^{+}$cells were irregularly aligned (Figure 5C). However, the neurotrophin receptor $\mathrm{P} 75^{\mathrm{NTR}}$ expression in inner pillar cells was not affected in the CKO (Figure 4D). The glutamate-aspartate transporter (GLAST) expression in inner border and inner phalangeal cells were detectable in the CKO cochlea, but they appeared to be disorganized and dislocated on the luminal layer (Figure 4E). The calcium-binding protein S100A marks inner HCs and surrounding inner border, inner phalangeal and inner pillar cells, as well as the Deiters' cells underlying the outer HCs (Figure 5E,F). S100A ${ }^{+}$cells were present in the $\mathrm{CKO}$, but they were disorganized with more than one cell on the luminal side. The $\mathrm{S}_{100 \mathrm{~A}^{+}}$cells on the luminal side where outer HCs are normally located were also Myo7a ${ }^{+}$cells in the base, but not in medial cochlear duct (Figure 5F). From

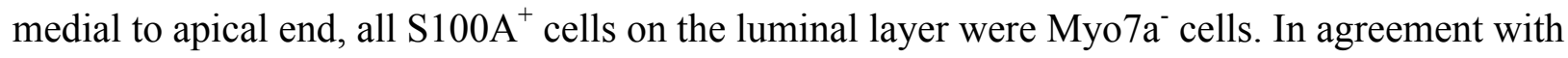
the calretinin staining (Figure 4C), the $\mathrm{S} 00 \mathrm{~A}^{+}$cells on the luminal layer likely represent the inner $\mathrm{HC}$ precursors that failed to differentiate into Myo $7 \mathrm{a}^{+}$cells. Nonetheless, our data indicate that Sox $2^{+} \mathrm{p} 27^{\mathrm{Kip} 1+} \mathrm{SCs}$ in the $\mathrm{Brg} 1^{\text {Cko/Cko }}$ failed to fully differentiate into Prox $1^{+} \mathrm{SCs}$, resulting in disorganized sensory epithelium.

In contrast to Sox 2 expression in the $\mathrm{CKO}$ cochlear sensory epithelium (Figure 5A,B), the expression of Eyal, which is normally expressed in differentiating hair cells at E17.5-18.5 
(Zou et al., 2008), was markedly reduced and only some faint signal was observed in the basal region (arrow, Figure 6A). Sixl is expressed in both hair and supporting cells (Zhang et al., 2017), but its expression in both cell types was almost undetectable in the CKO cochlea (Figure 6A). In addition, Atohl expression was undetectable in the CKO (Figure 6A). This indicates that in the absence of Eya1/Six1, Sox 2 alone is insufficient to induce Atoh1 expression in Sox $2^{+}$ progenitors to promote a $\mathrm{HC}$ fate in vivo.

\section{Eya1-Six1 simultaneously interacts with the BAF60a/c structural subunit}

The SWI/SNF complexes contain more than 10 associated BAF factors, which generate a variety of subcomplexes involved in dynamic and tissue-specific interactions with TFs and other chromatin-modifying enzymes. Some BAFs have variants that are selectively used in cell fate commitment (Ho and Crabtree, 2010). Peptides from BAF170, BAF155 and BAF60b were detected in our proteomics analysis of the Eyal complexes purified from HEK293 cells that constitutively express a multi-tagged-Eya1 (2×HA-3×Flag-Eya1) (J. Xu et al., manuscript in preparation), leading to the hypothesis that Eyal activity may depend on the activity of such BAFs. We therefore examined whether Eyal interacts with such BAFs in the cochlea. We have generated the $2 \times$ HA-3 $\times$ Flag-Eya1 knockin mouse line (J. Li et al., manuscript in preparation), which allows us to use anti-Flag or -HA for immunoprecipitating the multi-tagged Eyal protein complexes. CoIP analyses using nuclear extracts from E13.5 cochleae confirmed physical association of Eya1 with Brg1, BAF170 and BAF155 (Figure 6B). BAF60 has three variants, a, $\mathrm{b}$ and $\mathrm{c}$, and in contrast to our mass spectrometry data showing interaction of Eyal with BAF60b in 293 cells, Eyal associated simultaneously with BAF60a/c but not BAF60b in the cochlea (Figure 6B). Consistent with our previous observation (Buller et al., 2001), Eyal formed a 
complex with Six1, which showed similar physical interaction with BAF60a/c but not with BAF60b (Figure 6-figure supplement 5A). In contrast, Brg1 physically associated with all three BAF60 variants along with BAF170/BAF155 (Figure 6B). This suggests formation of specific BAF60a/c-based SWI/SNF-Eya1/Six1 complexes.

We next investigated their expression pattern during cochlea development. BAF60a showed higher levels of expression in the prosensory primordium (Figure 6C,D) and later in both HCs and SCs (Figure 6-figure supplement 5B), while BAF60c appeared to be expressed in discrete cells in the prosensory domain (Figure 6E) and by E18.5 its expression was only detected in the HCs and pillar cells Figure 6-figure supplement 5C). Both BAF60a/c are also expressed in Hensen's cells flanking the outer HCs and in SGNs (Figure 6-figure supplement 5B,C). In Brg1 ${ }^{C k o / C k o}$ cochlea at E14.0-14.5 (Tam at E11.5), both BAF60a/c levels were reduced specifically in the Eyal-expressing domain (GER and prosensory primordium) (Figure 6D,E). However, in contrast to marked depletion of Brg1 in the prosensory progenitors (Figure 1-figure supplement $1 \mathrm{G}$ ), some $\mathrm{BAF} 60 \mathrm{a}^{+}$cells and clusters of $\mathrm{BAF} 60 \mathrm{c}^{+}$discrete cells were detectable in the CKO prosensory primordium. While it is possible that other ATPase-based SWI/SNF complexes containing BAF60a/c may form in different subsets of the progenitors, our data suggest that in the absence of Brg1-BAF complex assembly, BAF60a/c may become unstable and undergo degradation.

Both BAF170/BAF155 were also detected in the prosensory primordium (Figure 6-figure supplement 5D,E). By E18.5, high levels of BAF170 were maintained in HCs and SCs and in GER as well as in stria vascularis and SGNs (Figure 6-figure supplement 5F). In contrast, BAF155 was weakly expressed in HCs and SCs but strongly in Henen's cells and LER (Figure 6-figure supplement 5G). Together, these data provide insight into how distinct BAF subunits 
might be used to generate a diversity of SWI/SNF complexes during cochlea morphogenesis to promote lineage-specific properties.

\section{Role for Brg1 in global gene activation in the cochlea}

To explore Brg1 genome-wide binding profile and its association with regulatory sequences in the prosensory progenitors, we performed ChIP-seq on E13.5 cochleae. MACS (Zhang et al., 2008) peak calling identified 8086 (with a $P$ value threshold of 1e-5) and 1292 (a $P$ value threshold of 1e-6) Brg1 peaks (Figure 7A). 28\% of Brg1-bound regions were within $5 \mathrm{~kb}$ of promoter-5' UTR and $\sim 72 \%$ were located within $5-500 \mathrm{~kb}$ of TSSs of the nearest gene (Figure 7B). Analysis of the $8086 \mathrm{Brg} 1$-enriched regions found 3079 ( 28\% promoter-proximal and $\sim 72 \%$ distal) associated with $\mathrm{H} 3 \mathrm{~K} 27 \mathrm{ac}$, a well characterized epigenetic mark associated with transcriptionally active chromatin (Figures 7A and Figure 7-figure supplement 6A). Consistent with Brg1 involvement in active and repressive gene expression in other tissues (Attanasio et al., 2014; Trotter and Archer, 2008), our cochlear ChIP-seq data indicate that Brg1 is associated not only with transcriptionally active chromatin, but also with other regulatory regions.

To explore whether Brg1-enriched sites contain consensus motifs and to reduce the number of regions for motif analysis, we examined the 1292 peaks for the presence of known motifs. The top three motifs contain consensus sequences for bZip proteins, Zinc finger proteins and SOX factors (Figure 7C), all of which are known to specifically interact with Brg1containing SWI/SNF and act as Brg1's transcriptional coregulators (King and Klose, 2017; Marathe et al., 2017; Trotter and Archer, 2008). Thus, this analysis provides insight into potential DNA-binding TFs that Brg1 interacts with at gene promoters and distal regulatory elements in the cochlea. 
Gene Ontology (GO) analysis showed highly enriched "biological process terms" near genes associated with processes involving development and morphogenesis, including inner ear and cochlea specific processes characteristic of cochlea development (Figure 7D and Figure 7figure supplement 6C). Brgl binding was evident at genes involved in cell division such as Mycn $(N m y c)$ (Figure 7E) and $M y c(c M y c)$ (Figure 7-figure supplement 6D) as well as at their downstream targets Cyclin D1/D2 (Figures 7E and Figure 7-figure supplement 6D). Genes related to inner ear/cochlea morphogenesis and development include $p 27^{\text {Kip } 1}$, TFs (such as Eya1/4, Six1/4, Sox2/4/5/9, Gata3, Sobp, Prox1, Id2/3, and Tbx18), and genes participating in Wnt signaling (Wnt5a/Fzd/Ptk7/Sfrp2/Cthrc1/Ptk7), Hh (Gli3/Ptch1), Fgf-signaling (Fgfr1/2/Fgf9), and Notch pathway (Jag1/Lfng/Dll1/Hes1/Hey1/Hey2/Notch1/2) (Supplementary files 1-3 and Figure 7-figure supplement 7). In contrast, no Brg1 peaks were identified at genes that are not expressed in the cochlea such as at Foxil (Figure 7E) and Six6 (Figure 7-figure supplement 6D) loci. Together, our ChIP-seq analysis not only indicates Brg1-binding of key neurosensory genes including Eya1, Six 1, and Sox 2 but also uncovers a robust set of Brg1-bound regions near genes involved in expected tissue-specific biological processes.

\section{ChIP assays confirm Brg1 binding at discrete regulatory regions in the Eya1, Six1, Sox2,}

\section{Eya4 and $p 27^{K i p 1}$ loci and co-occupancy by BAF60a/c}

At the Eyal locus, Brg1-bound sites were recovered in promoter (I) and intergenic (II) regions and most of these regions are ECRs (evolutionary conserved regions) based on comparison of mouse with human genomes (Figure 8A). Since Eyal expression in the otocyst also requires Brg1 activity, we speculated that Brg1 may bind to the same elements at the Eyal locus to activate its expression in early otic progenitors and to maintain its expression in cochlear sensory 
epithelium. ChIP-qPCR assays on otocysts dissected from E10.5 embryos confirmed strong Brg1 enrichment at the promoter ( +610 to $+429 \mathrm{bp} ;+2$ to $-180 \mathrm{bp} ;-373$ to $-532 \mathrm{bp}$ ) (primer positions, Supplementary file 4) in wild-type samples (Figure 8B). For the intergenic sites, ChIP-qPCR revealed strong association of Brg1 with an ECR (-913924 to -914124 bp), but very weak enrichment at two non-ECRs (-196849 to -197024 bp and -197523 to $-197695 \mathrm{bp})$ that had relatively weaker Brgl signals in ChIP-seq (red stars). ChIP-qPCR on Eyal ${ }^{\text {CreERT2 }}$; Brgl $1^{\text {flfl }}$ littermates showed a significant reduction in Brg1-enrichments at these regions (Figure 8B). Similarly, ChIP-qPCR for four regions at the Sixl locus confirmed Brgl binding to two of the downstream intergenic elements $(+12227$ and +8299$)$ and disruption of Brg1 association with these regions in Brgl CKO otocysts (Figure 8-figure supplement 8A,D). These results further support that Brg1-occupancy is required for the activity of these genes during inner ear development.

At the Sox2 locus, Brg1-binding was recovered at an intergenic region located at $\sim 691 \mathrm{~kb}$ upstream from the promoter (Figure 8-figure supplement 8B). This region was also recovered in the Brg1 ChIP-seq data on E11.5 forebrain (Attanasio et al., 2014). ChIP-qPCR within $1 \mathrm{~kb}$ of the Brg1-bound region (asterisks, Figure 8-figure supplement 8B) confirmed strong Brg1 binding at +691335 and +191656 in wild-type otocyts and large reduction of Brg1 binding at these sites in Brgl CKO (Figure 8-figure supplement 8E), thus suggesting possible requirement of Brg1occupancy at this region for Sox2 expression in the otic progenitors. ChIP assay also confirmed Brg1-binding at the Eya4 gene (Figure 8-figure supplement 8C,F), which is known to have a role in sensory cell development as EYA4-deficiency in humans causes hearing impairment (Pfister et al., 2002).

To characterize if BAF60a or BAF60c is a component of the SWI/SNF complex binding 
at the Eyal locus, we performed ChIP assay with anti-BAF60a or BAF60c respectively. BAF60a showed association to other three Brg1-bound sites (+610, +2 and -193924; Figure 8B), while BAF60c revealed enrichment at all four sites with different affinity levels. Brgl-deficiency led to $\sim 2$ to 4-fold reduction in BAF60a or BAF60c enrichment at these sites. The co-occupancy patterns of BAF60a/c on Brg1-bound sites could result from transient binding by separate SWI/SNF components in different subsets of Eya $1^{+}$cells. In summary, the ChIP experiments on E10.5 otocysts confirm enrichment of Brg1 at the discrete ECR regions in the Eyal locus from early stage. Furthermore, our results support that Brg1 is the preferred ATPase subunit of the SWI/SNF complex binding at the discrete regulatory regions of Eyal to regulate its expression.

The cyclin-dependent kinase inhibitor $\mathrm{p} 27^{\mathrm{Kip} 1}(\mathrm{Cdkn1b})$ is required to correctly time cell cycle exit of the prosensory progenitors prior to their differentiation and is regulated by Sox 2 in cochlear SCs (Liu et al., 2012). Brg1 ChIP-seq recovered strong peaks at the discrete promoterproximal and distal regions in the p27kipl locus (Figure $8 \mathrm{C}$ ). We compared these regions with public Sox2 ChIP-seq data from immortalized Sox $2^{+}$otic progenitor cells (Kwan et al., 2015) and found that Sox2-bound sites at the promoter and distal sites overlap with the Brg1-bound regions (Figure 8C), suggesting that Sox2 may interact with Brg1-BAF complexes to regulate $p 27^{\text {Kip } 1}$ expression. Indeed, Sox 2 physically interacts with Brg1 in the cochlea by coIP analysis (Figure 6-figure supplement 5A). ChIP assays on E13.5 cochleae confirmed association of Brg1 to the promoter-proximal and distal sites $(-519,-277,+1753,+6513,+6983$ and +7955$)$ (Figure 8C,D). Brgl-deficiency (given Tam from E11.5) significantly decreased Brg1 binding at these sites. Thus, reduced p27 $7^{\mathrm{Kip} 1}$ expression in Brgl CKO cochlea (Figure 3) is strongly associated with decreased $\mathrm{Brg} 1$ binding to the discrete regulatory regions of $p 27^{\text {Kip } 1}$ gene. $\mathrm{Brg} 1$-deficincy also led to decreased BAF60a or BAF60c enrichment at these Brg1-bound sites. Together, these 
co-occupancy patterns at the ECRs further support that Brg1-based SWI/SNF complex containing BAF60a or BAF60c is necessary for $p 27^{\text {Kipl }}$ expression through binding to the discrete regulatory regions in the $p 27$ Kip1 locus.

\section{Discussion}

We have previously shown that Eya1-Six1 interacts with Brg1-BAFs to convert cochlear nonsensory GER cells to SGNs, yet nothing is known about the in vivo requirement of the Brg1BAF complexes for inner ear neurosensory cell fate commitment and their involvement in regulatory pathways controlling inner ear formation. Here we demonstrate that otic ectoderm requires the chromatin-remodeling enzyme $\mathrm{Brg} 1$ to form inner ear structures. The pattern of cochlear Brg1 genomic binding and its strong association with $\mathrm{H} 3 \mathrm{~K} 27 \mathrm{ac}$ suggest that $\mathrm{Brg} 1$ is substantially involved in regulatory pathways governing inner ear development.

Ablation of Brg1 in Eyal-expressing cells blocked the activation of neurosensory cell fate inducing programs. This phenotype is linked to loss of Eyal/Sixl expression, which results in loss of Sox 2 and Neurog1 expression. Eya1, Six 1 and Sox 2 form the core transcription circuitry for neurosensory precursor cell fate specification and subsequent differentiation in the inner ear. However, to date, nothing is known about what lies upstream of these genes in the inner ear. Our findings provide the first in vivo evidence that Brg1-based SWI/SNF chromatin remodeling factors regulate the expression of Eyal, Six 1 and Sox 2 at different stages of inner ear development. Our data show that Brg1 plays a crucial role in coordinating multiple processes during otic neurosensory cell specification and differentiation.

How do the Brg1-based SWI/SNF complexes help to establish and maintain neurosensory cell identities? SWI/SNF chromatin remodeling complexes are typically recruited 
to genomic sites via physical interactions with sequence-specific TFs to execute spatiotemporal control over specific target genes during developmental processes (Ho and Crabtree, 2010; Neely et al., 1999). Therefore, Brg1 may differentially impact the spatial and temporal expression of Eya1/Six1 in the otic ectoderm. The otic placode forms from a common pre-placodal ectoderm, which is defined by the members of Eya and Six families. However, unlike Six 1/4 and Eya2 that are coexpressed in the pre-placodal ectoderm, the mouse Eyal is expressed in the otic and epibranchial placodal ectodermal regions (Chen et al., 2009). Thus, Eyal may have a specific role in defining an otic fate from the pre-placodal ectoderm and the timing of Eyal activation may require a unique Brg1-SWI/SNF complex in response to external signals. In support of this, ChIP assays on E10.5 otocysts confirmed Brg1 association with the promoter and distal regulatory regions at the Eyal locus. Future work is required to reveal exactly how the binding of Brgl to Eyal locus is related to establishing otic fate. This process likely involves other TFs that are expressed in the ectoderm, during the transition from the pre-placodal stage to Eya $1^{+}$ multipotent otic progenitors before neurosensory cell fate induction.

Once Eya1 is activated, Brg1-BAFs may alter the structure of chromatin at lineagespecific genes to facilitate the function of Eya1, which interacts with Six 1 and other factors. Our observation of a direct physical interaction between Brg1-BAFs and Eya1-Six1 suggests that Eya1-Six1 use the chromatin-remodeling properties of Brg1-BAF complexes to regulate transition from a multipotent otic ectodermal cell to a prosensory restricted progenitor cell by activating lineage-specific genes such as Sox 2 or to a committed neuronal fate by activating Neurog1-Neurod1. This explains why ablation of both Eya1/Six1 also leads to a lack of Sox2 (Figure 2) or Neurog1-Neurodl (Ahmed et al., 2012b) expression. Since the expression of Eyal/Six 1 is markedly reduced in Brgl CKO, lack of Brg1-SWI/SNF binding at these genes 
could prevent their activation. This in turn simultaneously prevents expression of Sox 2 and Neurog1-Neurod1 and results in a developmental block of neurosensory cell fate specification. We thus speculate that Brg1 may bind to the regulatory elements at the Eyal and Sixl loci in order to activate their coexpression before neurosensory fate induction in otic placode and to maintain the coexpression of these genes in otic ectoderm and cochlear sensory epithelium at later stages. Future studies using a combination of genomic and molecular approaches are required to reveal the molecular details of TFs and Brg1-BAF complexes involved in activating Eyal or Six 1 during early otic fate induction.

Our data also show an essential role for Brg1 in both cell survival and proliferation within the otic ectoderm, which is consistent with Brgl's necessity in cell survival and proliferation in various tissues. As Eya1 or Six 1 is also required for normal cell proliferation and survival in the otic ectoderm (Zheng et al., 2003; Zou et al., 2006), Brg1 may cooperate with Eya1/Six 1 to regulate these processes, which explains why loss of any of these genes causes a decrease in cell number in the otic ectoderm and leads to growth arrest. Ablation of Brg1 in the developing cochlea at later stages underscores the continuous contribution of Brg1-BAF remodelers during prosensory precursor cell differentiation, from the regulation of precursor proliferation and survival to the induction of cell type-specific genes.

In gain-of-function studies, we previously demonstrated the requirement of Brg1 activity and its interaction with Eya1/Six1 in inducing a robust neuronal differentiation program from cochlear nonsensory GER cells or ectodermal cells surrounding the otocyst in explants (Ahmed et al., 2012b). However, it remained unknown whether Brg1 is also necessary for HC fate induction. The present loss-of-function study now demonstrates the necessity of Brg1 in inducing auditory sensory epithelium formation. Brg1 is not only essential for specification of 
the prosensory domain but also for terminal differentiation of precursor cells within the domain into either HCs or SCs. Conditional deletion of Brg1 between E11.75-12.5 prevented Sox2 ${ }^{+}$ prosensory progenitors from becoming $\mathrm{p} 27^{\mathrm{Kip} 1+}$ postmitotic precursors. Thus, Brg1-BAF complexes may facilitate regulation of $p 27^{\text {Kip } 1}$ expression and other downstream genes by Sox2, thus explaining why Sox 2 alone is insufficient to induce $\mathrm{p} 27^{\mathrm{Kip} 1}$ expression. This is consistent with the physical interaction between Sox 2 and Brg1 and Sox2 co-occupancy at a subset of Brg1-bound regions at the $p 27^{\text {Kip } 1}$ locus. The necessity of Brg1 activity in $p 27$ Kip 1 regulation is further supported by the marked reduction in Brg1 enrichment at discrete regulatory ECRs in the p27Kipl locus in the Brgl CKO cochlea (Figure 8D). Interestingly, when Brg1 was deleted at later stages between E13.5-14.0 after $p 27^{k i p 1}$ activation, $\mathrm{p} 27^{\mathrm{Kip} 1+}$ cells also failed to fully differentiate into Myo7a ${ }^{+} \mathrm{HCs}$ or Prox $1^{+}$SCs. Although this developmental defect may have resulted from a failure to appropriately regulate chromatin structure, it may also be attributed to a deficiency in modification of other proteins that are crucial for HC fate induction or SC differentiation, such as Eya1, Six1, Atoh1 and Prox1, all of which are not expressed in the Brg1 mutant. In the absence of Eyal and Six1 in Brg1 CKO cochlea, Sox2 alone appears to be incapable of activating Atoh1 or maintaining Prox 1 expression in Sox $2^{+}$precursors to promote HC fate induction or SC differentiation. Although it is currently unclear whether Eya1-Six1 also use Brg1 activity to facilitate Atohl activation and HC induction during cochlea development, in our ChIP-seq data sets, weak enrichment of Brg1 and H3K27ac was observed at the $1.4 \mathrm{~kb}$ Atoh1 HC-specific enhancer located $\sim 3.4 \mathrm{~kb} \mathrm{3'}$ ' of the Atoh1 coding sequence (red star, Figure S8). This enhancer mediates transcriptional regulation by Eya1-Six1-Sox2 (Ahmed et al., 2012a). It is possible that Atoh1 may not be activated yet in the cochlear samples collected from E13.5 embryos for our ChIP-seq, as suggested by the weak H3K27ac signals at this enhancer. This is 
consistent with the observation that Atohl activation is induced between E13.5-14.5 (Chen et al., 2002). We are currently performing Brg1 ChIP-seq on cochleae at later stages between E14.5 to E15.5 to examine if Brg1 enrichment at this HC-specific Atoh1 enhancer increases. Stronger Brg1 binding at later stages would suggest that recruitment of Brg1-BAF complex to the $1.4 \mathrm{~kb}$ HC-specific enhancer may confer temporal control of HC fate activation by Eya1-Six1-Sox2. Nonetheless, stronger Brg1 enrichment at the promoter-proximal regions of the Atoh1 suggests that Brg1 may bind to discrete regulatory regions of the Atohl to temporally control its initial activation in Sox $2^{+}$progenitors and upregulation during $\mathrm{HC}$ fate commitment. Brg1-enrichment at the Proxl locus but not $p 75$ locus further indicates the essential function of Brg1-based chromatin remodeling in the activation of transcriptional programs in distinct subtypes of precursor cells during sensory epithelium development (Figure 7-figure supplement 7). Future work is necessary to elucidate how Brg1 interacts with other factors, including Eya1, Six1 and Sox2, to target its binding sites at the Sox $2, p 27^{\text {Kip } 1}$ and Atohl loci identified from our ChIP-seq data using a combination of transgenic, genomic and molecular approaches.

Our results suggest that Brg1-BAFs may have cell type-specific and context-dependent roles in inner ear development. During development, distinct BAF subunits interact with specific TFs, recruiting the complex to the sites of action of the corresponding factors to control distinct transcriptional programs (Ho et al., 2009; Lessard et al., 2007; Wang et al., 1996). Simultaneous interactions of Eya1/Six1 with BAF60a/c and BAF170/155 suggests formation of separate SWI/SNF complexes containing distinct BAF subunits in different subsets of Eya1- or Six1expressing cells, in response to differentiation cues, that activate a diverse program of lineagespecific gene expression. This is in an agreement with the differential and overlapping patterns of BAF170/155/60a/c in the cochlear sensory epithelium. As BAF60a or BAF60c acts in 
recruiting TFs (Chen et al., 2012; Oh et al., 2008) and nuclear receptors (Hsiao et al., 2003) to the SWI/SNF chromatin remodeling complex, the variants a/c of the BAF60 structural subunit may recruit Eya1-Six 1 to the SWI/SNF complexes to regulate stage-dependent and cell typespecific programs. In response to signals, the alternative usage of specific SWI/SNF variants by the prosensory progenitors may direct distinct differentiation programs. The selective assembly of SWI/SNF with BAF60c and BAF170 may activate HC-specific genes, while Brg1 along with BAF60a-Eya1 and BAF60a-Six 1 may promote both $\mathrm{HC}$ and $\mathrm{SC}$ gene activation programs during terminal differentiation. The observation of a unique pattern of BAF170 expression in the pillar cells suggests that distinct subtypes of SCs may also require cell type-specific SWI/SNF complexes. We speculate that during otic development and differentiation, otic-specific activators may both recruit and require chromatin-remodeling activities for stable binding to the regulatory regions of otic- and neurosensory-specific genes. Future molecular studies will be required to elucidate if these complexes are recruited by transient interactions with different TFs including Eya1, Six1, and Sox2, and whether association of Eya1-Six1 with BAF60a/c recruits Eya1-Six1 into a Brg1-based SWI/SNF complex that is able to remodel chromatin and activate neurosensory-specific gene transcription.

In summary, our results indicate that Brg1 activity is required for Eyal/Six1 expression and that Eya1-Six1 may use chromatin remodeling properties of the Brg1-BAFs to fine-tune control of neuronal or sensory cell fate determination. Activation of Sox 2 in the otic ectoderm requires both Eya1/Six1 function. However, in vivo, Sox2 alone without Brg1 activity or Eya1/Six 1 activity is insufficient to induce $p 27^{\text {Kip } 1}$ or Atoh 1 expression in the Sox $2^{+}$progenitors in the cochlea. These findings illustrate how chromatin-remodeling enzymes are intricately involved in the transcriptional control of the master regulators of development and the 
differentiation of neurosensory cells in the inner ear. Since ChIP-seq failed to recover Brg1 enrichment at Neurogl or Neurod1 loci, how chromatin remodeling of the Brg1-BAFs intersects with chromatin remodeling of these neuronal cell-specific bHLH TFs remains to be demonstrated. In conclusion, the genome-wide maps of in vivo Brg1 binding sites generated in this study provide a foundation for understanding cochlea-specific genome-wide changes in chromatin structure driven by this key chromatin remodeling factor, which is critical to understanding the role of Brg1 in development and disease. This study provides not only a novel framework for understanding how TFs might direct neurosensory cell commitment and differentiation but also implies possibilities for therapeutic intervention by targeting the SWI/SNF complex in regeneration.

\section{Methods}

\section{Animals, genotyping and tamoxifen administration}

All animal protocols were approved by Animal Care and Use Committee of the Icahn School of Medicine at Mount Sinai (protocol \#06-0807).

Brgl $^{f l}$ (Sumi-Ichinose et al., 1997), Eyal-CreERT2 (Xu et al., 2017; Xu et al., 2014), Sox2-CreERT2 (Arnold et al., 2011), and Eyal ${ }^{+/-}$;Six1 ${ }^{+/-}$(Zheng et al., 2003) mice were maintained on a 129/Sv and C57BL/6J mixed background at the Icahn School of Medicine at Mount Sinai Animal Facility. Mice were bred using timed mating, and noon on the day of vaginal plug detection was considered as E0.5. For induction of the CreERT2 protein, tamoxifen (Sigma, T5648) was dissolved in corn oil (Sigma, C8267) and administrated (1.5 mg/10 g body weight) by oral gavage. Observed variations among Brgl mutants is likely due to pre-existing developmental variation between embryos when tamoxifen was given. 


\section{Histology, immunostaining, and in situ hybridization (ISH)}

Histological examination, whole-mount and section immunostaining and ISH were carried out according to standard procedures. Briefly, inner ears were fixed in $4 \%$ paraformaldehyde (PFA) for $1 \mathrm{hr}$ at $4^{\circ} \mathrm{C}$, dehydrated, and embedded in wax or OCT. Paraffin or frozen sections were generated at $6 \mu \mathrm{m}$. For ISH, tissues were fixed overnight. We used five or six embryos for each genotype at each stage for each probe and the result was consistent in each embryo.

For immunostaining, sections were stained with primary antibodies listed below. Cy2-, Cy5- and FITC-conjugated secondary antibodies were used. Hoechst 3342 was used for nuclear staining.

Primary antibodies: anti-Brg1 (ab110641, Abcam), -BAF170 (sc-17838, Santa Cruz), BAF155 (sc-48350, Santa cruz), -BAF60a (sc-135843, Santa Cruz), -BAF60b (sc-101162, Santa Cruz), -BAF60c (ab171075, Abcam), -Sox2 (PA1-094, Thermo Fisher), -Myo7a (25-6790,

Proteus and 138-1-s, DSHB), -p27 kip1 (554069, BD Pharmingen), -Calretinin (MA5-14540, Thermo Fisher), -p75 ${ }^{\mathrm{NTR}}$ (\#07-476, EMD Millipore), -S100A (ab11428, Abcam), -GLAST (ab416, Abcam), -Prox1 (AB5475, Millipore), -Brg1 (sc-10768, Santa Cruz), -BAF170 (sc166237, Santa Cruz), Cy3-, Cy2-, Cy5- and FITC-conjugated secondary antibodies were used. Hoechst 3342 was used for nuclear staining.

\section{EdU and TUNEL Assays}

The EdU assay was performed using a kit (catalog no. C10640, Life Technologies) following the manufacturer's instructions. EdU was co-injected with tamoxifen at 9 am of E11.5 and embryos were harvested at afternoon of E13.5. EdU was also injected at 9 am of E9.5 embryos following 
tamoxifen treatment at 9 am of E8.0-8.5 and embryos were harvested 2 hours after EdU injection at noon of E9.5. The TUNEL assay was performed using the Apop Tag kit for in situ apoptosis fluorescein detection (catalog no. NC9815837, Millipore) following the manufacturer's instructions.

\section{Cell counts and calibration}

EdU-incorporated cells and Hoechst-stained nuclei in E9.5 otocyst were counted respectively. EdU-incorporated Sox $2^{+}$prosensory progenitors in the floor of the cochlear epithelium were counted in the entire cochlea. Values represent average number of $\mathrm{EdU}^{+}$cells or proliferation rate of $\mathrm{EdU}^{+}$cells/total Hoechst ${ }^{+}$cells ( \pm standard deviations) per otocyst or $\mathrm{EdU}^{+} \mathrm{Sox}^{+}$cells ( \pm standard deviations) per section $(6 \mu \mathrm{m})$ or per cochlea. 15 sections per cochlea and 6 cochleae for each sample were measured. Two-tailed Student's $t$-test was used for statistical analysis.

\section{Co-immunoprecipitation}

Embryonic cochleae (E13.5-14.5) were homogenized and lysed in $10 \mathrm{mM}$ HEPES, pH7.5, 1.5 $\mathrm{mM} \mathrm{MgCl2}, 10 \mathrm{mM} \mathrm{KCl}, 1 \mathrm{mM}$ dithiothreitol (DTT) and protease and phosphatase inhibitors cocktail. After removal of cytoplasmic fraction, the crude nuclei pellet was lysed in nuclear extraction buffer (20 mM mM HEPES, pH7.5, $1.5 \mathrm{mM} \mathrm{MgCl2,} 420 \mathrm{mM} \mathrm{NaCl}, 0.2 \mathrm{mM}$ EDTA, 25\% glycerol, $1 \mathrm{mM}$ dithiothreitol (DTT) and protease and phosphatase inhibitors cocktail). The extracted nuclear proteins were diluted in IP buffer (Tris-Cl, $\mathrm{pH} 8.0,100 \mathrm{mM} \mathrm{NaCl}, 0.1 \%$ NP-40, $10 \%$ glycerol). The lysates were pre-cleared with protein A/G beads (sc-2003, Santa Cruz). After removal of the beads, the lysates were incubated with $\sim 1 \mu \mathrm{g}$ primary antibodies anti-Brg1 (ab110641, Abcam), anti-Flag (F1804, Sigma) or -anti-Six1 (HPA001893; Sigma) with rotation 
at $4{ }^{\circ} \mathrm{C}$, overnight. Lysates were incubated with Protein $\mathrm{A} / \mathrm{G}$ beads to precipitate complexes for 3 $\mathrm{h}$ at $4{ }^{\circ} \mathrm{C}$ with rotation. The immunocomplexes were recovered by centrifugation and washed three times with IP buffer. SDS buffer was added to the precipitate and boiled for 5 min. Proteins were size separated in SDS-PAGE. The gels were blotted onto an Immobilon-P membrane (IPVH00010, Millipore), blocked with 5\% non-fat dry milk and incubated with the previously described antibodies. HRP-conjugated secondary antibodies (Goat anti-Mouse $\operatorname{IgG}(\mathrm{H}+\mathrm{L})$, A4416, Sigma; Goat anti-Rabbit IgG $(\mathrm{H}+\mathrm{L}), 31460$, Thermo Scientific) were used for detection using the enhanced chemiluminescence (ECL) method (WBKLS0500, Millipore).

\section{ChIP-seq and ChIP-qPCR}

Cochleae were dissected from E13.5-14.0 embryos and cross-linked with 1\% formaldehyde at room temperature for 30 mins. Samples were then homogenized and lysed in cold lysis buffer (50 mM HEPES-KOH, pH 7.5, 140 mM NaCl, 1 mM EDTA, 10\% glycerol, 0.5\% NP-40, 0.25\% Triton $\mathrm{X}-100,1 \times$ protease inhibitors) and gently rocked at $4^{\circ} \mathrm{C}$ for 10 minutes in $15 \mathrm{ml}$ conical tubes. Cells were pelleted at $2000 \mathrm{~g}$ at $4^{\circ} \mathrm{C}$ and resuspended in cold wash buffer $(10 \mathrm{mM}$ TrisHCl, pH 8.0, $200 \mathrm{mM} \mathrm{NaCl}, 1 \mathrm{mM}$ EDTA, $0.5 \mathrm{mM}$ EGTA, 1× protease inhibitors) and gently rocked at $4^{\circ} \mathrm{C}$ for 10 minutes in $15 \mathrm{ml}$ conical tubes. Cells were pelleted at $2000 \mathrm{~g}$ at $4^{\circ} \mathrm{C}$ in a table top centrifuge and resuspended in $1 \mathrm{ml}$ cold sonication buffer $(10 \mathrm{mM}$ Tris-Cl, $\mathrm{pH} 8.0,2$ mM EDTA, 0.1\% SDS) and sonicated to 200-500 bp fragments using a Covaris S220 Focusedultrasonicator. Sonicated chromatin was cleared by pelleting insoluble material at 13,000 RPM at $4^{\circ} \mathrm{C}$ followed by preclear with protein $\mathrm{A} / \mathrm{G}$ beads and incubation with $1 \sim 2 \mu \mathrm{g}$ antibody overnight (anti-Brg1, ab110641, Abcam; anti H3K27ac, ab4792, Abcam). Next, Protein A/G beads (20 $\mu \mathrm{l})$ were added to the lysate and incubated at $4^{\circ} \mathrm{C}$ for $5 \mathrm{hrs}$. Immunoprecipitated material was 
washed with low salt buffer (20 mM Tris-Cl 8.0, $150 \mathrm{mM} \mathrm{NaCl}, 2 \mathrm{mM}$ EDTA, 1\% Triton X-100, 0.1\% SDS), high salt buffer (20 mM Tris-Cl pH 8.0, $500 \mathrm{mM} \mathrm{NaCl}, 2 \mathrm{mM}$ EDTA, 1\% Triton X100, 0.1\% SDS), and LiCl wash buffer (10 mM Tris-HCl pH 8.0, 250 mM LiCl, 1 mM EDTA, $1 \%$ NP-40, 1\% sodium deoxycholate) and one time with TE plus $\mathrm{NaCl}$, followed by elution and reverse crosslinking overnight at $65^{\circ} \mathrm{C}$. The quality controls of ChIPed DNA was performed with Qubit 2.0 Fluoremeter using dsDNA HS assay Kit (Q32854, ThermoFisher Scientific) and Agilent 2200 TapeStation System using Hightivity D1000 Reagenets (5067-5585, Agilent). The libraries for sequencing were prepared with the ThruPlex DNA-seq Kit (R400429, Rubicon Genomics) and sequenced on Illumina HiSeq 2500 system.

For ChIP-qPCR, chromatin derived from embryonic otocysts or from E13.5 cochleae and IgG, anti-Brg1 (ab110641, Abcam), anti-BAF60a antibody (ab224229, Abcam), or anti-BAF60c (ab171075, Abcam) was used for ChIP respectively. The ChIPed DNAs were subjected to quantitative PCR (qPCR) amplification with StepOnePlus PCR system and SYBR green PCR Master Mix kit (4309155, Applied Biosystems). The enrichment fold of IP over mock IP was calculated using the comparative $\mathrm{Ct}$ (threshold cycle) method. Data was normalized with inputs and the enrichment of mock IP was considered 1-fold. This experiment was repeated three times and each qPCR was performed in triplicate. The primers used for ChIP-qPCR are listed in Supplementary Table 4. The DNA positions are denoted relative to the transcriptional start site $(+1)$.

\section{Peak calling and gene otology analysis}

The ChIP-seq data were first checked for quality using the various metrics generated by FastQC (v0.11.2) (http://www.bioinformatics.babraham.ac.uk/projects/fastqc). Raw sequencing reads 
were then aligned to the mouse mm10 genome using default settings of Bowtie (v2.2.0)

(Langmead et al., 2009). Only uniquely-mapped reads were retained and duplicates were

removed. Peak-calling was performed using MACS (v2.1.1) (Zhang et al., 2008) with various p-

value cutoffs as reported in the main text. Motif enrichment analysis was performed using the

Homer package (v4.8.3) (Heinz et al., 2010). The peak annotation and gene ontology analysis

was performed using GREAT program (McLean et al., 2010) and Panther classification system

(Thomas et al., 2003). The conservation between mouse and human genome sequences was

analyzed online with ECR Brower (Ovcharenko et al., 2004).

Accession numbers. The ChIP-seq data reported in this paper were submitted to GEO

(GSE119545).

\section{Acknowledgements}

We thank L. Zhang for technical assistance. This work was supported by the NIH RO1

DC014718 (PXX) and NYSTEM C029566 (PXX).

\section{Competing interests}

The authors declare no competing interests.

\section{Author contributions}

J.X., J.L., Y.H.E.L, A.R., T.Z., H.J., data collection and analysis, validation, writing-

review/editing; L.S., B.F., data analysis, writing-review/editing; P.X. X., conceptualization, supervision, funding acquisition, data analysis, writing-original draft, writing-review/editing. All authors approved the final version of the manuscript. 


\section{Reference}

Abdelhak, S., Kalatzis, V., Heilig, R., Compain, S., Samson, D., Vincent, C., Weil, D., Cruaud, C., Sahly, I., Leibovici, M., et al. (1997). A human homologue of the Drosophila eyes absent gene underlies branchio-oto-renal (BOR) syndrome and identifies a novel gene family. Nat Genet $15,157-164$.

Ahmed, M., Wong, E.Y., Sun, J., Xu, J., Wang, F., and Xu, P.X. (2012a). Eya1-Six1 interaction is sufficient to induce hair cell fate in the cochlea by activating Atoh1 expression in cooperation with Sox2. Dev Cell 22, 377-390.

Ahmed, M., Xu, J., and Xu, P.X. (2012b). EYA1 and SIX1 drive the neuronal developmental program in cooperation with the SWI/SNF chromatin-remodeling complex and SOX2 in the mammalian inner ear. Development 139, 1965-1977.

Arnold, K., Sarkar, A., Yram, M.A., Polo, J.M., Bronson, R., Sengupta, S., Seandel, M., Geijsen, N., and Hochedlinger, K. (2011). Sox2(+) adult stem and progenitor cells are important for tissue regeneration and survival of mice. Cell Stem Cell 9, 317-329.

Attanasio, C., Nord, A.S., Zhu, Y., Blow, M.J., Biddie, S.C., Mendenhall, E.M., Dixon, J., Wright, C., Hosseini, R., Akiyama, J.A., et al. (2014). Tissue-specific SMARCA4 binding at active and repressed regulatory elements during embryogenesis. Genome Res 24, 920-929.

Buller, C., Xu, X., Marquis, V., Schwanke, R., and Xu, P.X. (2001). Molecular effects of Eya1 domain mutations causing organ defects in BOR syndrome. Hum Mol Genet 10, 2775-2781. Bultman, S., Gebuhr, T., Yee, D., La Mantia, C., Nicholson, J., Gilliam, A., Randazzo, F., Metzger, D., Chambon, P., Crabtree, G., et al. (2000). A Brg1 null mutation in the mouse reveals functional differences among mammalian SWI/SNF complexes. Mol Cell 6, 1287-1295. Chen, B., Kim, E.H., and Xu, P.X. (2009). Initiation of olfactory placode development and neurogenesis is blocked in mice lacking both Six1 and Six4. Dev Biol 326, 75-85.

Chen, L., Fulcoli, F.G., Ferrentino, R., Martucciello, S., Illingworth, E.A., and Baldini, A. (2012). Transcriptional control in cardiac progenitors: Tbx1 interacts with the BAF chromatin remodeling complex and regulates Wnt5a. PLoS Genet 8, e1002571.

Chen, P., Johnson, J.E., Zoghbi, H.Y., and Segil, N. (2002). The role of Math1 in inner ear development: Uncoupling the establishment of the sensory primordium from hair cell fate determination. Development 129, 2495-2505.

Danielian, P.S., Muccino, D., Rowitch, D.H., Michael, S.K., and McMahon, A.P. (1998). Modification of gene activity in mouse embryos in utero by a tamoxifen-inducible form of Cre recombinase. Curr Biol 8, 1323-1326.

Hagstrom, S.A., Pauer, G.J., Reid, J., Simpson, E., Crowe, S., Maumenee, I.H., and Traboulsi, E.I. (2005). SOX2 mutation causes anophthalmia, hearing loss, and brain anomalies. Am J Med Genet A 138A, 95-98.

Hargreaves, D.C., and Crabtree, G.R. (2011). ATP-dependent chromatin remodeling: genetics, genomics and mechanisms. Cell Res 21, 396-420.

Heinz, S., Benner, C., Spann, N., Bertolino, E., Lin, Y.C., Laslo, P., Cheng, J.X., Murre, C., Singh, H., and Glass, C.K. (2010). Simple combinations of lineage-determining transcription factors prime cis-regulatory elements required for macrophage and B cell identities. Mol Cell 38, 576-589. 
Ho, L., and Crabtree, G.R. (2010). Chromatin remodelling during development. Nature 463, 474-484.

Ho, L., Jothi, R., Ronan, J.L., Cui, K., Zhao, K., and Crabtree, G.R. (2009). An embryonic stem cell chromatin remodeling complex, esBAF, is an essential component of the core pluripotency transcriptional network. Proc Natl Acad Sci U S A 106, 5187-5191.

Hsiao, P.W., Fryer, C.J., Trotter, K.W., Wang, W., and Archer, T.K. (2003). BAF60a mediates critical interactions between nuclear receptors and the BRG1 chromatin-remodeling complex for transactivation. Mol Cell Biol 23, 6210-6220.

Kiernan, A.E., Pelling, A.L., Leung, K.K., Tang, A.S., Bell, D.M., Tease, C., Lovell-Badge, R., Steel, K.P., and Cheah, K.S. (2005). Sox2 is required for sensory organ development in the mammalian inner ear. Nature 434, 1031-1035.

King, H.W., and Klose, R.J. (2017). The pioneer factor OCT4 requires the chromatin remodeller BRG1 to support gene regulatory element function in mouse embryonic stem cells. Elife 6.

Kwan, K.Y., Shen, J., and Corey, D.P. (2015). C-MYC transcriptionally amplifies SOX2 target genes to regulate self-renewal in multipotent otic progenitor cells. Stem Cell Reports 4, 4760.

Langmead, B., Trapnell, C., Pop, M., and Salzberg, S.L. (2009). Ultrafast and memoryefficient alignment of short DNA sequences to the human genome. Genome biology 10, R25. Lessard, J., Wu, J.I., Ranish, J.A., Wan, M., Winslow, M.M., Staahl, B.T., Wu, H., Aebersold, R., Graef, I.A., and Crabtree, G.R. (2007). An essential switch in subunit composition of a chromatin remodeling complex during neural development. Neuron 55, 201-215.

Liu, Z., Walters, B.J., Owen, T., Brimble, M.A., Steigelman, K.A., Zhang, L., Mellado Lagarde, M.M., Valentine, M.B., Yu, Y., Cox, B.C., et al. (2012). Regulation of p27Kip1 by Sox2 maintains quiescence of inner pillar cells in the murine auditory sensory epithelium. J Neurosci 32, 10530-10540.

Marathe, H.G., Watkins-Chow, D.E., Weider, M., Hoffmann, A., Mehta, G., Trivedi, A., Aras, S., Basuroy, T., Mehrotra, A., Bennett, D.C., et al. (2017). BRG1 interacts with SOX10 to establish the melanocyte lineage and to promote differentiation. Nucleic Acids Res 45, 6442-6458.

McLean, C.Y., Bristor, D., Hiller, M., Clarke, S.L., Schaar, B.T., Lowe, C.B., Wenger, A.M., and Bejerano, G. (2010). GREAT improves functional interpretation of cis-regulatory regions. Nature biotechnology 28, 495-501.

Muchardt, C., Bourachot, B., Reyes, J.C., and Yaniv, M. (1998). ras transformation is associated with decreased expression of the brm/SNF2alpha ATPase from the mammalian SWI-SNF complex. EMBO J 17, 223-231.

Neely, K.E., Hassan, A.H., Wallberg, A.E., Steger, D.J., Cairns, B.R., Wright, A.P., and Workman, J.L. (1999). Activation domain-mediated targeting of the SWI/SNF complex to promoters stimulates transcription from nucleosome arrays. Mol Cell 4, 649-655.

Oh, J., Sohn, D.H., Ko, M., Chung, H., Jeon, S.H., and Seong, R.H. (2008). BAF60a interacts with p53 to recruit the SWI/SNF complex. J Biol Chem 283, 11924-11934.

Ovcharenko, I., Nobrega, M.A., Loots, G.G., and Stubbs, L. (2004). ECR Browser: a tool for visualizing and accessing data from comparisons of multiple vertebrate genomes. Nucleic acids research 32 , W280-W286. 
Ozaki, H., Nakamura, K., Funahashi, J., Ikeda, K., Yamada, G., Tokano, H., Okamura, H.O., Kitamura, K., Muto, S., Kotaki, H., et al. (2004). Six1 controls patterning of the mouse otic vesicle. Development 131, 551-562.

Pfister, M., Toth, T., Thiele, H., Haack, B., Blin, N., Zenner, H.P., Sziklai, I., Nurnberg, P., and Kupka, S. (2002). A 4-bp insertion in the eya-homologous region (eyaHR) of EYA4 causes hearing impairment in a Hungarian family linked to DFNA10. Mol Med 8, 607-611.

Ruf, R.G., Xu, P.X., Silvius, D., Otto, E.A., Beekmann, F., Muerb, U.T., Kumar, S., Neuhaus, T.J., Kemper, M.J., Raymond, R.M., Jr., et al. (2004). SIX1 mutations cause branchio-oto-renal syndrome by disruption of EYA1-SIX1-DNA complexes. Proc Natl Acad Sci U S A 101, 80908095.

Sumi-Ichinose, C., Ichinose, H., Metzger, D., and Chambon, P. (1997). SNF2beta-BRG1 is essential for the viability of F9 murine embryonal carcinoma cells. Mol Cell Biol 17, 59765986.

Thomas, P.D., Campbell, M.J., Kejariwal, A., Mi, H., Karlak, B., Daverman, R., Diemer, K., Muruganujan, A., and Narechania, A. (2003). PANTHER: a library of protein families and subfamilies indexed by function. Genome research 13, 2129-2141.

Trotter, K.W., and Archer, T.K. (2008). The BRG1 transcriptional coregulator. Nucl Recept Signal 6, e004.

Wang, W., Xue, Y., Zhou, S., Kuo, A., Cairns, B.R., and Crabtree, G.R. (1996). Diversity and specialization of mammalian SWI/SNF complexes. Genes Dev 10, 2117-2130.

$\mathrm{Xu}$, J., Ueno, H., Xu, C.Y., Chen, B., Weissman, I.L., and Xu, P.X. (2017). Identification of mouse cochlear progenitors that develop hair and supporting cells in the organ of Corti. Nat Commun 8, 15046.

Xu, J., Wong, E.Y., Cheng, C., Li, J., Sharkar, M.T., Xu, C.Y., Chen, B., Sun, J., Jing, D., and Xu, P.X. (2014). Eya1 interacts with Six2 and Myc to regulate expansion of the nephron progenitor pool during nephrogenesis. Dev Cell 31, 434-447.

Xu, P.X., Adams, J., Peters, H., Brown, M.C., Heaney, S., and Maas, R. (1999). Eya1-deficient mice lack ears and kidneys and show abnormal apoptosis of organ primordia. Nat Genet 23, 113-117.

Zhang, T., Xu, J., Maire, P., and Xu, P.X. (2017). Six1 is essential for differentiation and patterning of the mammalian auditory sensory epithelium. PLoS Genet 13, e1006967. Zhang, Y., Liu, T., Meyer, C.A., Eeckhoute, J., Johnson, D.S., Bernstein, B.E., Nusbaum, C., Myers, R.M., Brown, M., Li, W., et al. (2008). Model-based analysis of ChIP-Seq (MACS). Genome Biol 9, R137.

Zheng, W., Huang, L., Wei, Z.B., Silvius, D., Tang, B., and Xu, P.X. (2003). The role of Six1 in mammalian auditory system development. Development 130, 3989-4000.

Zou, D., Erickson, C., Kim, E.H., Jin, D., Fritzsch, B., and Xu, P.X. (2008). Eya1 gene dosage critically affects the development of sensory epithelia in the mammalian inner ear. Hum Mol Genet 17, 3340-3356.

Zou, D., Silvius, D., Fritzsch, B., and Xu, P.X. (2004). Eya1 and Six1 are essential for early steps of sensory neurogenesis in mammalian cranial placodes. Development 131, 55615572.

Zou, D., Silvius, D., Rodrigo-Blomqvist, S., Enerback, S., and Xu, P.X. (2006). Eya1 regulates the growth of otic epithelium and interacts with Pax2 during the development of all sensory areas in the inner ear. Dev Biol 298, 430-441. 
bioRxiv preprint doi: https://doi.org/10.1101/434159; this version posted October 3, 2018. The copyright holder for this preprint (which was not certified by peer review) is the author/funder, who has granted bioRxiv a license to display the preprint in perpetuity. It is made available under aCC-BY 4.0 International license. 


\section{Figure legends}

\section{Figure 1. Deletion of Brg1 using Eya1 ${ }^{C r E R T 2}$ in the otic placode results in growth arrest and} lack of inner ear structures. Tam was given from E8.5-9.0. (A-D) Anti-Brg1 staining in otic placode (A), otocyst (B), and developing cochlea (C,D). (E) H\&E stained otocyst of wild-type and Eyal ${ }^{C r e E R} ; B r g l^{f l f l}(C k o / C k o)$ littermates. (F) H\&E stained inner ear sections of E15.5 wildtype and $C k o / C k o$ littermates. Arrows indicate cavity-like structures. (G) EdU and (I) TUNEL assays. $(\mathrm{H}, \mathrm{J})$ Statistic analysis of $\mathrm{EdU}^{+}$or $\mathrm{TUENL}^{+}$cells from each otocyst. Data refer to the average of 3 embryos per genotype. $P$-value was calculated for $+/+$ and $B r g 1^{C k o / C k o}$ using Twotailed Student's $t$-test. Abb.: CD, cochlear duct; OC, organ of Corti; OV, otic vesicle; SPG, spiral ganglion; TB, temple bone. Scale bars: $50 \mu \mathrm{m}$ (A-E, G,I), $200 \mu \mathrm{m}$ (F).

Figure supplement 1. Immunostaining confirms selective deletion of Brg1 in the otocyst and cochlear epithelium using Eya1 ${ }^{\text {CreERT2 }}$.

Figure 2. Brg1 specifies neurosensory fates in the otocyst. $(A-H)$ Whole-mount $(A, B, G, H)$ and section (C-F) ISH for Eya1, Six1, Sox2, Dlx5, Neurog1 and Neurod1 in otocyst of wild-type or Brgl $1^{\text {Cko/Cko }}$ littermates or (I-L) for Sox 2 and Dlx 5 in wild-type and Eya1;Six 1-null embryos. Lines in $\mathrm{K}$ indicate regions of sections shown on the right panels. Abb.: d, dorsal; v, ventral. Scale bars: $350 \mu \mathrm{m}(\mathrm{A}, \mathrm{B}, \mathrm{G}, \mathrm{H}), 50 \mu \mathrm{m}(\mathrm{C}-\mathrm{F}, \mathrm{I}-\mathrm{L})$.

Figure supplement 2. Conditional deletion of Sox2 in the otic placode does not alter the pattern of Dlx5, Eya1 and Six1 expression.

Figure 3. Brg1 specifies the prosensory domain in the cochlea. (A-D) Sox $2 / \mathrm{p} 27^{\mathrm{Kip} 1}$ staining of E14.5 whole-cochlea (Tam at E12.0-12.5 (A-C) or at E11.75-12.5 (D) or of cochlear sections (E- 
H). Lower panels of E-H are stained with H\&E. Arrows point to p2 $7^{\mathrm{Kip} 1+}$ cells in the apex (B-D) or reduced Sox 2 in cells on the luminal surface in the sensory domain (E-H). Scale bars: $200 \mu \mathrm{m}$ (A-D), $30 \mu \mathrm{m}(\mathrm{E}-\mathrm{H})$.

Figure supplement 3. Brg1 is necessary for normal cell proliferation and survival in the cochlear sensory epithelium.

Figure 4. Brg1 is necessary for $\mathrm{HC}$ fate induction. (A) Myo7a staining of whole-cochlea at E18.0 (Tam at E13.5-14.0). (B) Higher magnification of boxed area in A. Arrows indicate disorganized Myo7a ${ }^{+}$cells. (C) Myo7a/Calretinin staining on sections of E18.5 cochlea. Lower panels stained with H\&E. Asterisks indicate misaligned cells in the organ of Corti. Abb.: IHC, inner HC; OHC, outer HC. Scale bars: $200 \mu \mathrm{m}(\mathrm{A}), 30 \mu \mathrm{m}(\mathrm{B}, \mathrm{C})$.

Figure supplement 4. Disruption of $\mathrm{HC}$ and SC differentiation in $\mathrm{Brg} \mathrm{C}^{\mathrm{Cko} / \mathrm{Cko}}$ cochlea.

Figure 5. Differentiation of supporting cells is disrupted in the absence of Brg1. Antibody labeling for $\mathrm{p} 27^{\mathrm{Kip} 1}$ (red) and Sox2 (green) (A,B), Prox1 (red) and Myo7a (green) (C), p75 ${ }^{\mathrm{NTR}}$ (green) and Myo7a (red) (D), GLAST (green) and S100A (red) (E), Myo7a (cyan) and S100A (red) (F) in the organ of Corti in wild-type and Brgl CKO $\left(\right.$ Eyal $\left.^{\mathrm{CreER}} ; \mathrm{Brgl}^{\mathrm{fl} f \mathrm{fl}}\right)$ littermates given tamoxifen from E13.5-14.0. Arrowheads in a point to Sox $2^{+}$but $\mathrm{p} 27^{\text {Kip1- }}$ cells in the GER, while arrows point to $\mathrm{p} 27^{\mathrm{Kip} 1+}$ but Sox $2^{-}$Hensen's cells. In the mutant, the organ of Corti appears narrower. Arrows in panel D point to cells positive for Myo7a and in G point to cells positive for both Myo7a and S100A. Scale bars: $30 \mu \mathrm{m}$.

Figure 6. Brg1 is necessary for maintenance of Eya1, Six1 and Atoh1 expression in the 
cochlear sensory epithelium and Eya1 selectively interacts with BAF60a/c but not BAF60b.

(A) In situ hybridization for Eyal, Sixl and Atoh1 in cochlea of wild-type or Brgl ${ }^{\text {Cko/Cko }}$

littermates at E17.5 given tamoxifen from E13.5. (B) CoIP analysis. Nuclear extracts were prepared from E13.5 cochleae. Antibodies for IP and western blot are indicated. (C,D)

Immunostaining for(C) BAF60a/Sox2 or (D) BAF60a alone on sections of wild-type cochlea at E14.0, Eyal ${ }^{\text {CreERT2 }}$ or Brgl $1^{C k o / C k o}\left(E y a 1^{C r e E R T 2} ;\right.$ Brgl $\left.^{f l / f l}\right)$ embryo at E14.5 given tamoxifen at E11.5-12.5. (E) Immunostaining for BAF60c in control $\left(E y a 1^{C r e E R T 2}\right.$ ) or Brg1 ${ }^{\text {Cko/Cko }}$ littermates given tamoxifen at E11.5-12.5. Scale bars: $200 \mu \mathrm{m}(\mathrm{A}), 50 \mu \mathrm{m}(\mathrm{C}-\mathrm{E})$.

Figure supplement 5. Brg1 physically interacts with Six1 and Sox2 and differential and overlapping patterns of BAF60a/c, BAF170 and BAF155 expression in the developing cochlea.

Figure 7. Genomic binding features of Brg1 in E13.5 cochlea. (A) Genomic distribution of Brg1-enriched regions and its overlap peaks with H3K27ac. (B) Distribution of Brg1 peaks relative to TSSs. (C) The top three motifs identified by Homer tools. (D) Differential enrichment for functional annotation terms associated Brg1-enriched regions. Shown are the top 10 enriched "Biological process terms" associated with the 1292 Brg1 enriched regions or specific to proximal Brg1 regions within $1 \mathrm{~kb}$ from promoters. (E) Genomic view of Brg1 enrichment at Mycn and Cyclin D1 loci but not at Foxil locus.

Figure supplement 6. Genomic binding features of Brg1 and H3K27ac in E13.5 cochlea. Figure supplement 7. Brg1 associates to both proximal and distal sites of key loci.

Figure 8. Brg1-bound regions at the Eya1 and p27Kip1 loci and co-occupancy patterns of 
BAF60a/c at these sites. (A,C) Brg1-bound regions at promoter-proximal (I) and distal (II) regions at the Eyal (A) or $p 27^{K i p 1}$ (C) loci and alignment of these regions with published Sox2 ChIP-seq (Kwan et al., 2015) and from mouse and human. Peak heights indicate degree of sequence homology; pink bars above the peaks denote ECRs; UTR (yellow) correspond thin blue box in IGV view; exons (thick blue boxes). Peak regions corresponding to ECRs (black asterisks) or non-ECRs (red asterisks) were selected for ChIP analysis. See Table S4 for positions of these sites. (B,D) ChIP-qPCR data using chromatin derived from otocysts of wildtype or $B r g 1^{C k o / C k o}$ littermates at E10.5 (Tam at E8.0-9.0) (B) or from cochleae of wild-type or Brgl $1^{\text {Cko/Cko }}$ littermates at E14.5 (Tam at E11.5-12.5) (D) and anti-Brg1, anti-BAF60a or antiBAF60c antibodies. Data were normalized with inputs and mock IgG control. Note very faint or no enrichment of Brg1 at the non-ECRs of Eyal, and weak enrichment for BAF60a at -373 bp of the Eyal and at +6983 bp and +7955 bp of the $p 27^{\text {Kipl }}$ and for BAF60c at +6983 bp of the $p 27^{\text {Kip } 1}$. Representative of three independent experiments, $\mathrm{n}=3$ for each group, $* P<0.01$, $* * P<0.05$ determined by Student's t-test).

Figure supplement 8. Brg1-bound regions at the Six1, Sox2 and Eya4 loci and ChIP assays confirm Brg1 enrichment at these loci. 

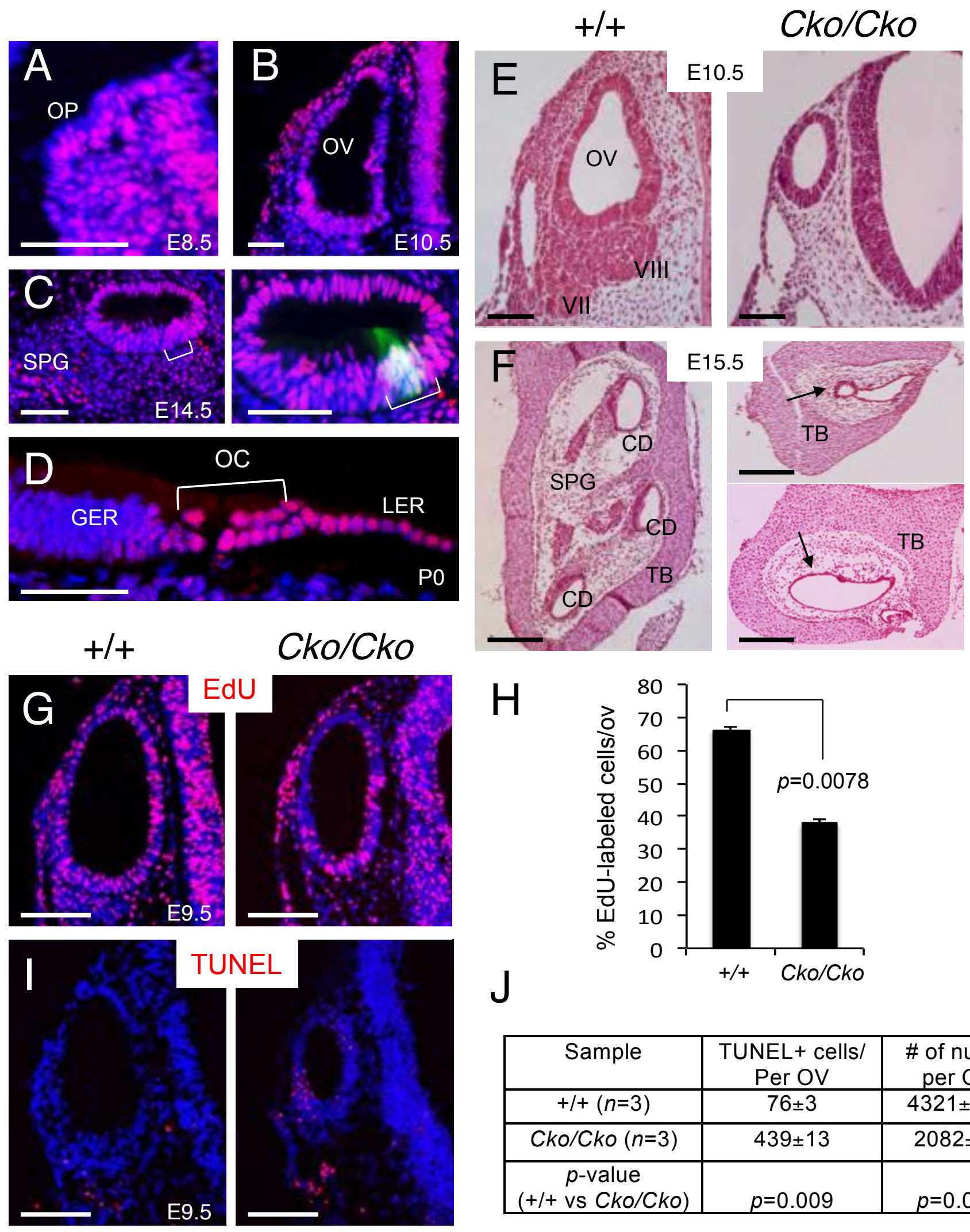

E15.5
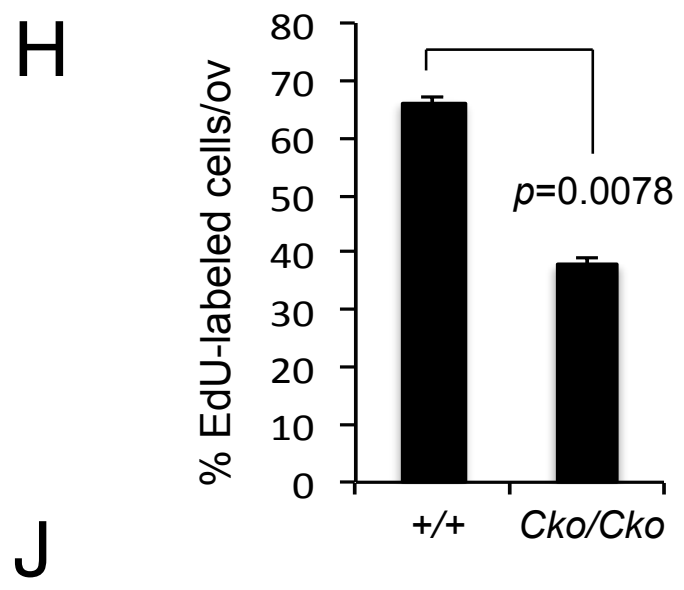

\begin{tabular}{|c|c|c|}
\hline Sample & $\begin{array}{c}\text { TUNEL+ cells/ } \\
\text { Per OV }\end{array}$ & $\begin{array}{c}\text { \# of nuclei } \\
\text { per OV }\end{array}$ \\
\hline$+/+(n=3)$ & $76 \pm 3$ & $4321 \pm 112$ \\
\hline Cko/Cko $(n=3)$ & $439 \pm 13$ & $2082 \pm 49$ \\
\hline $\begin{array}{c}p \text {-value } \\
(+/+ \text { vs Cko/Cko })\end{array}$ & $p=0.009$ & $p=0.007$ \\
\hline
\end{tabular}




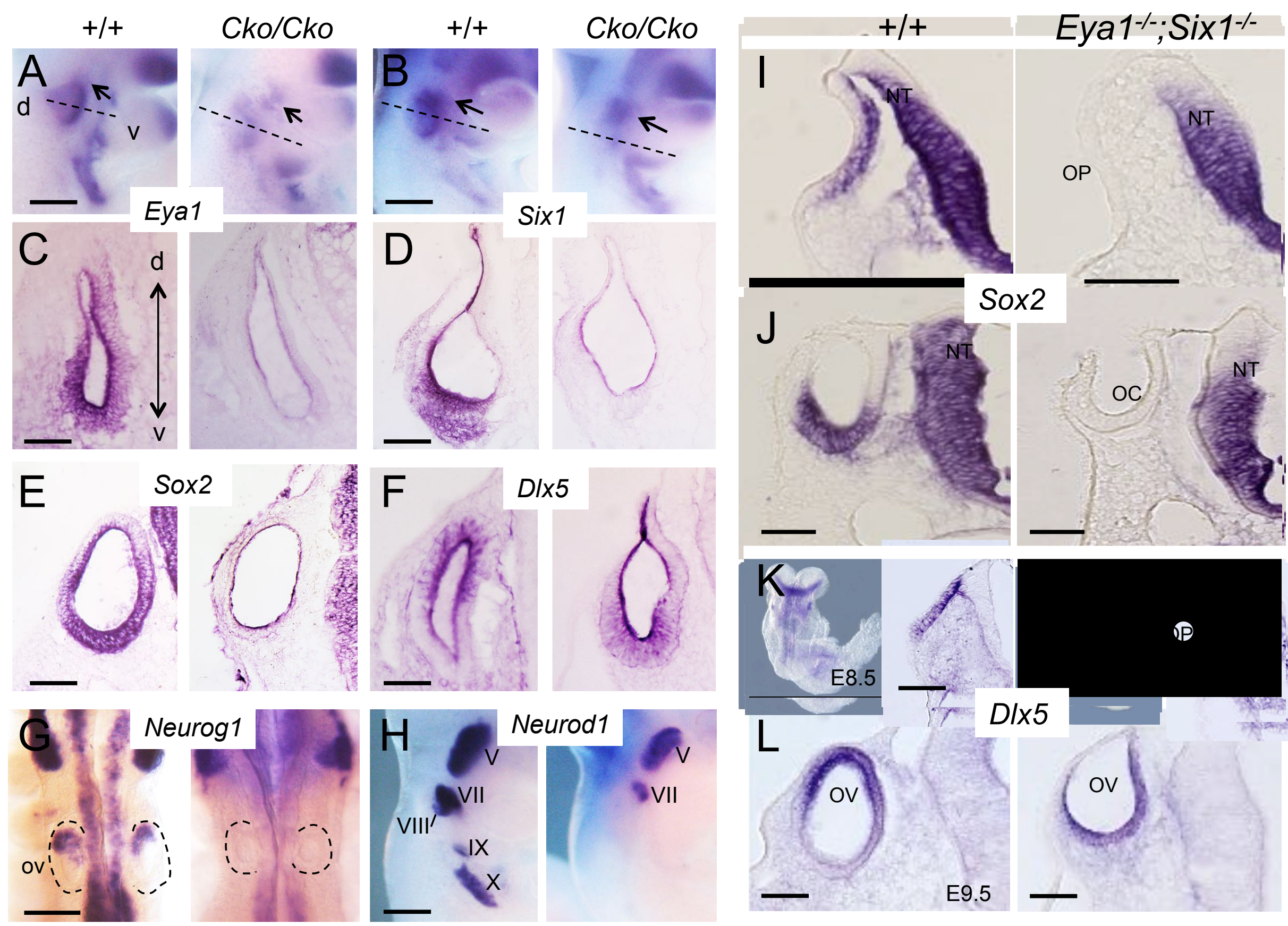




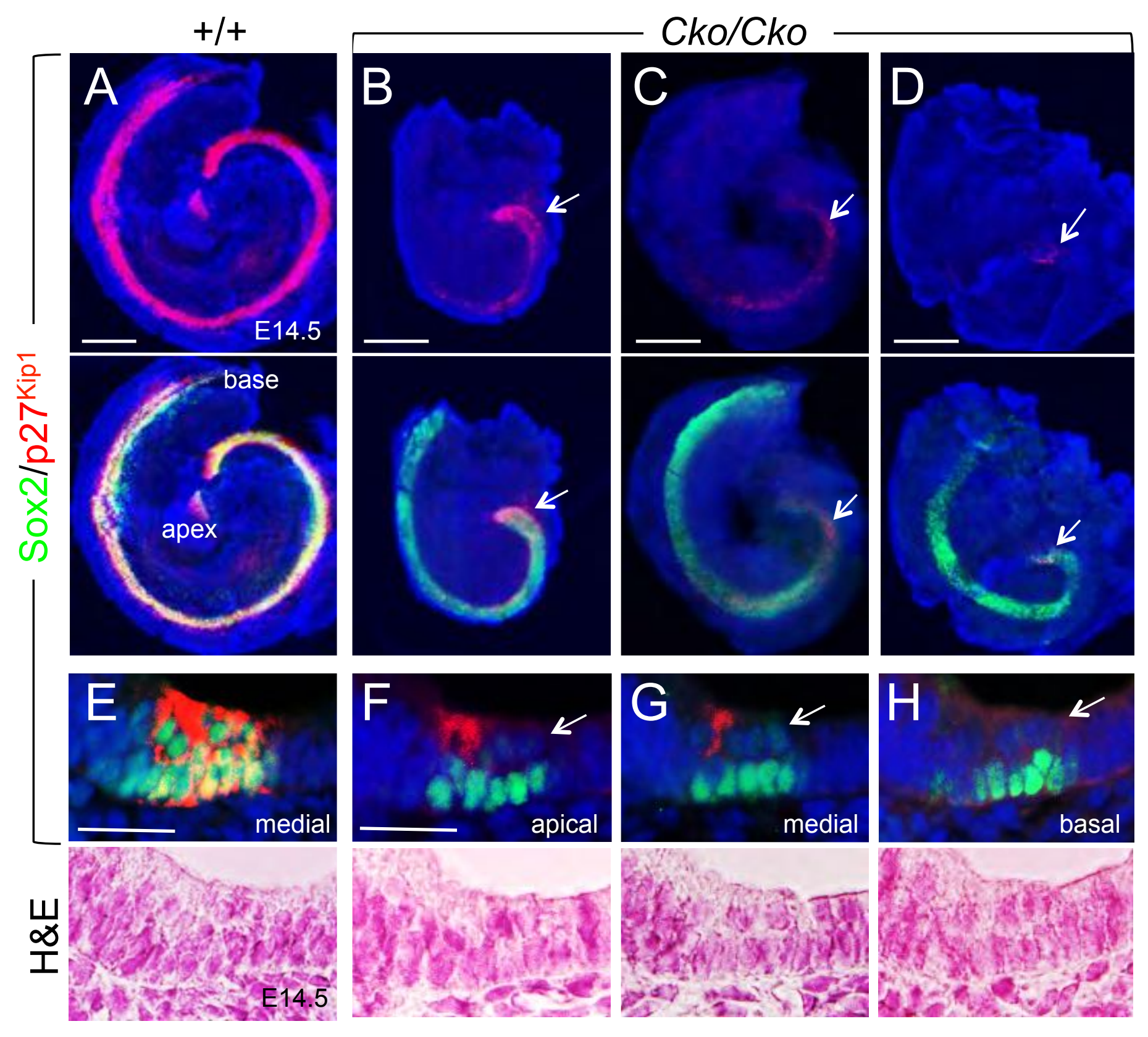




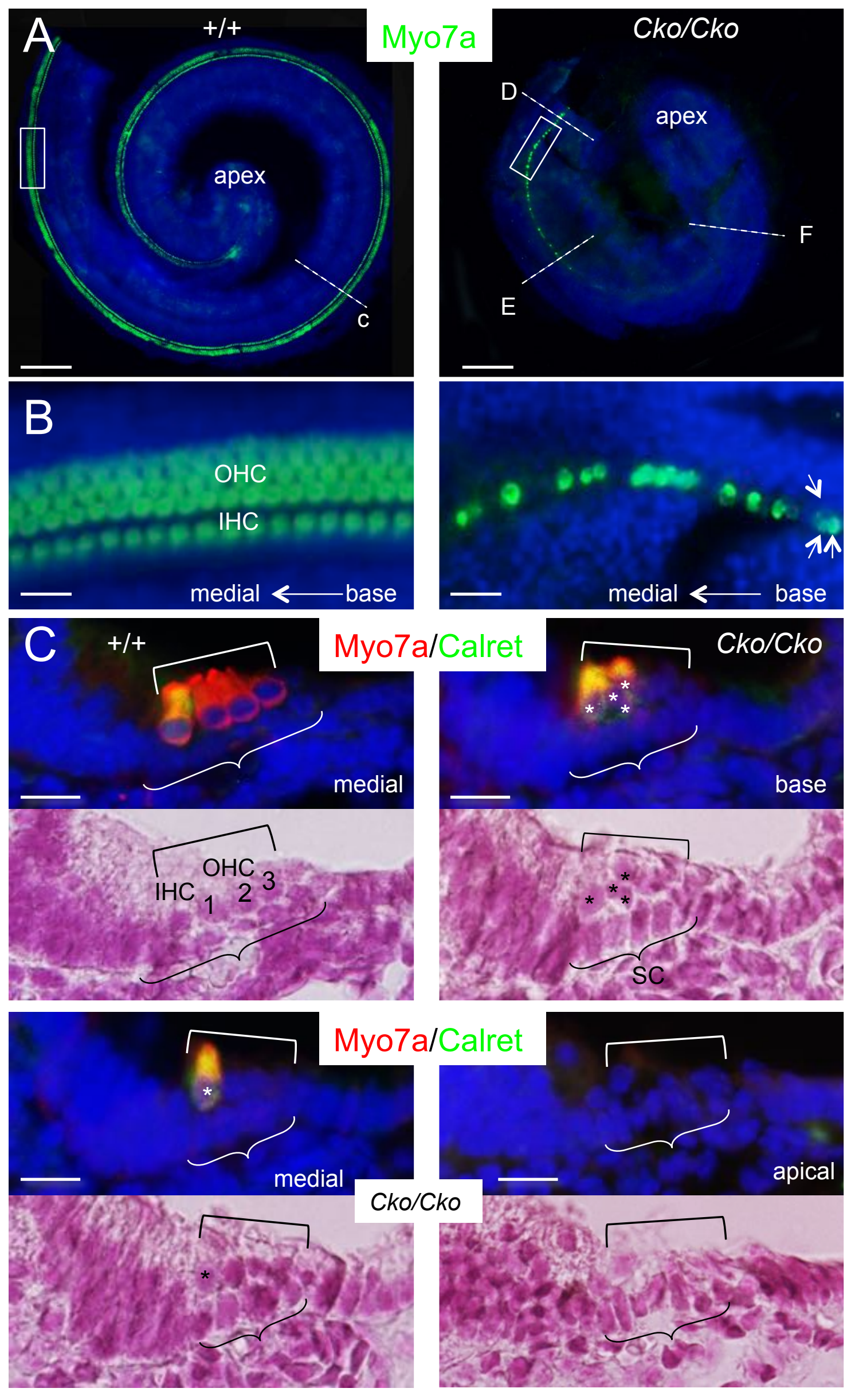



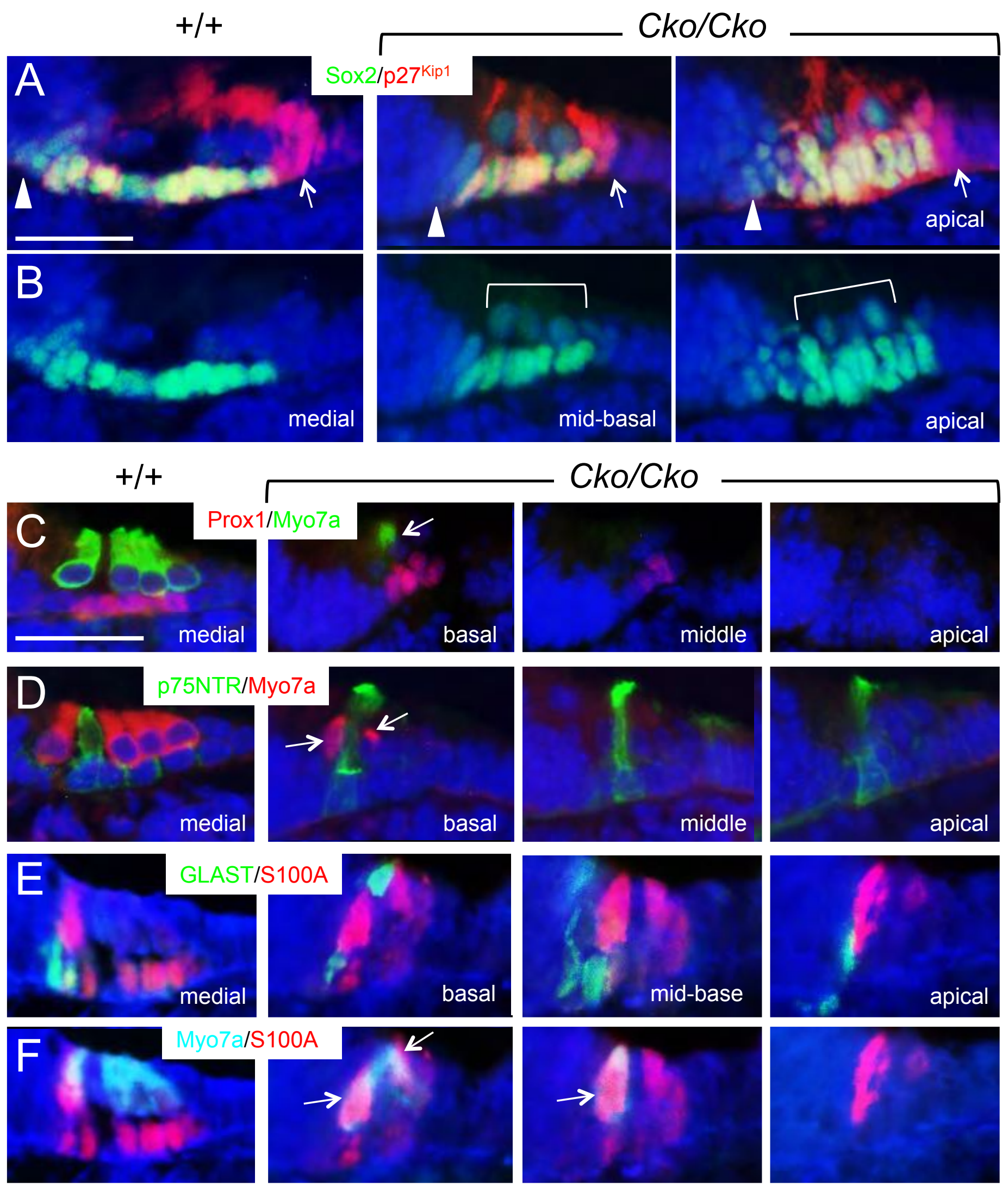
A

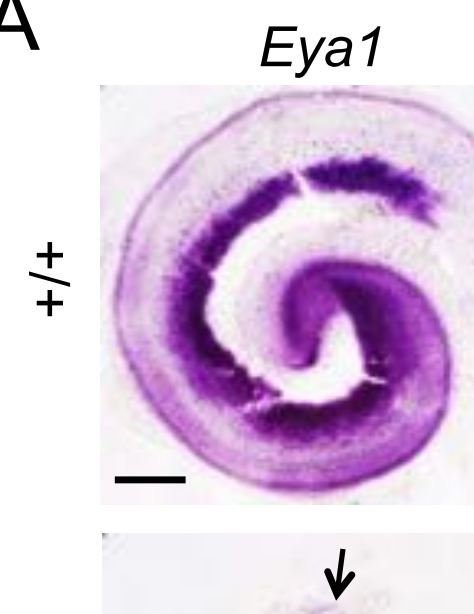

$\frac{0}{0}$
$\frac{y}{0}$
$\frac{1}{0}$
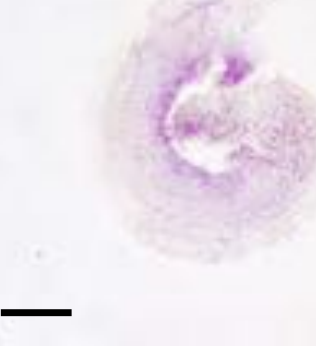

C
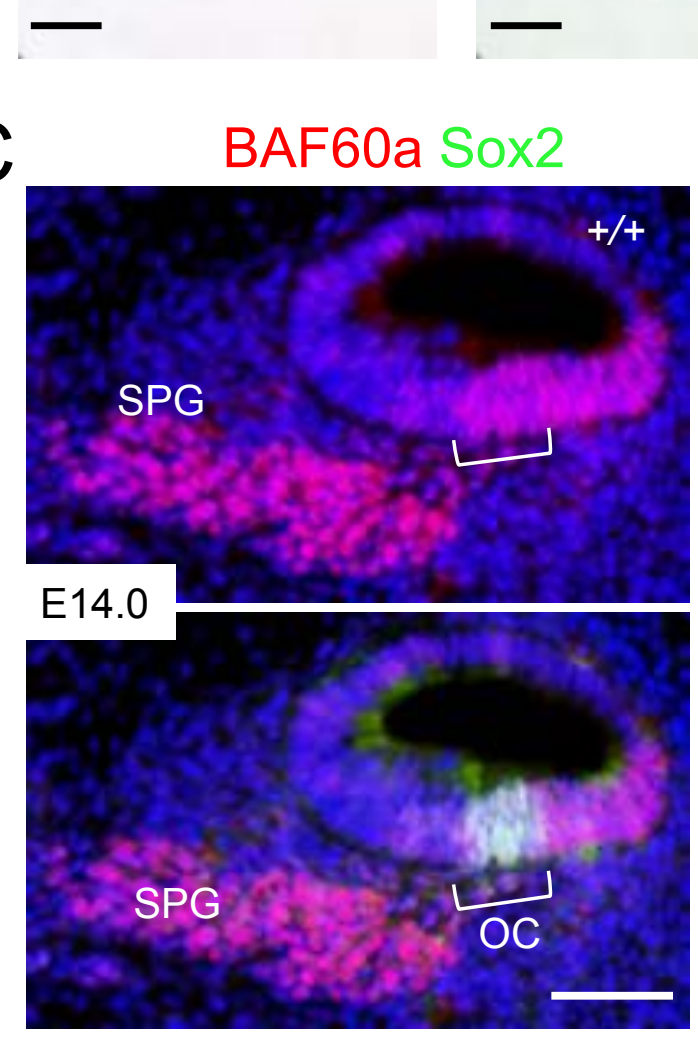

Six 1

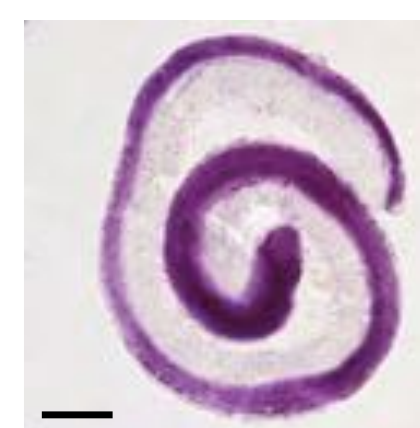

-

D

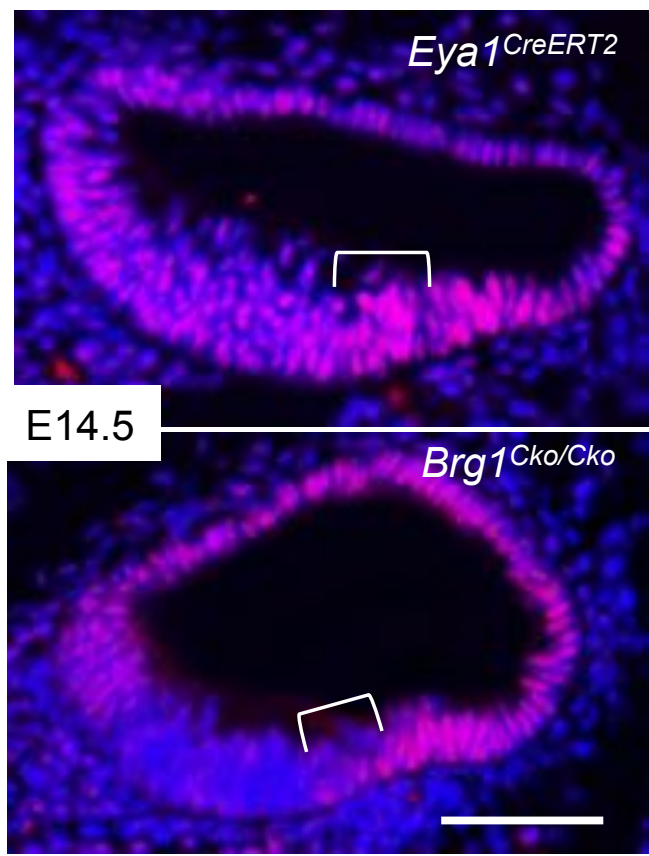

B ColP analysis

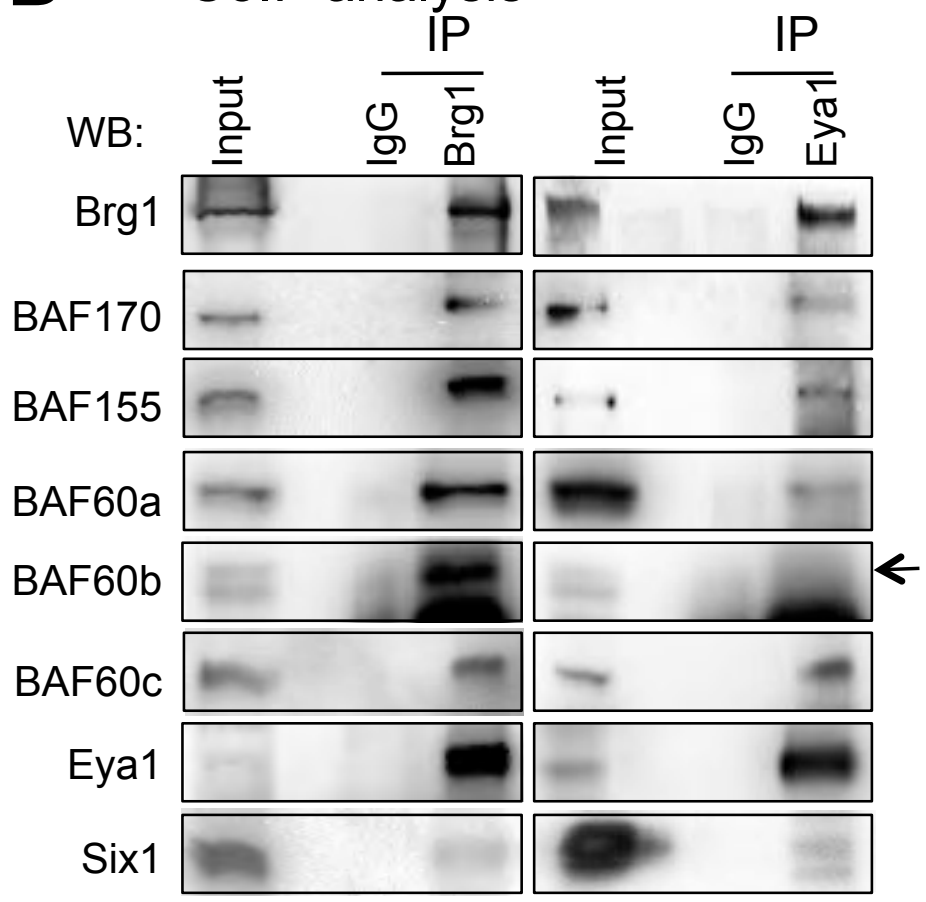

E BAF60c

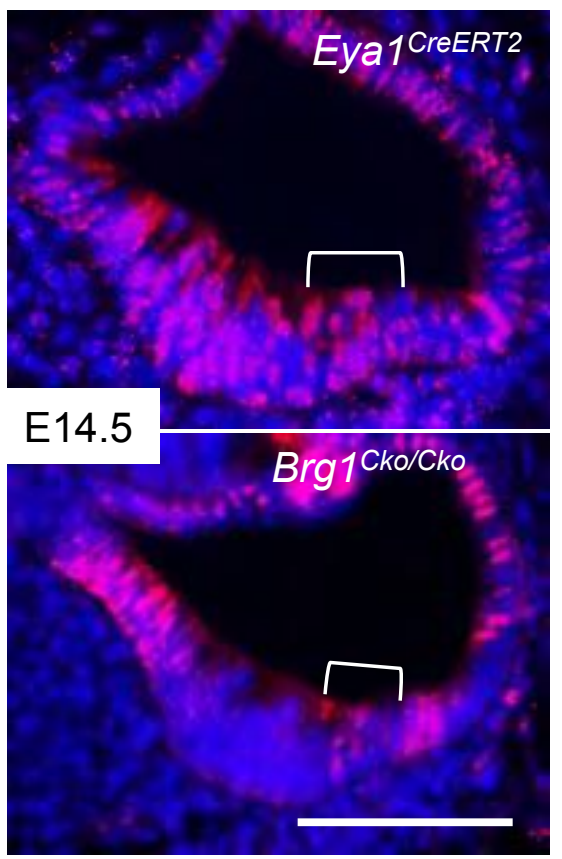


A

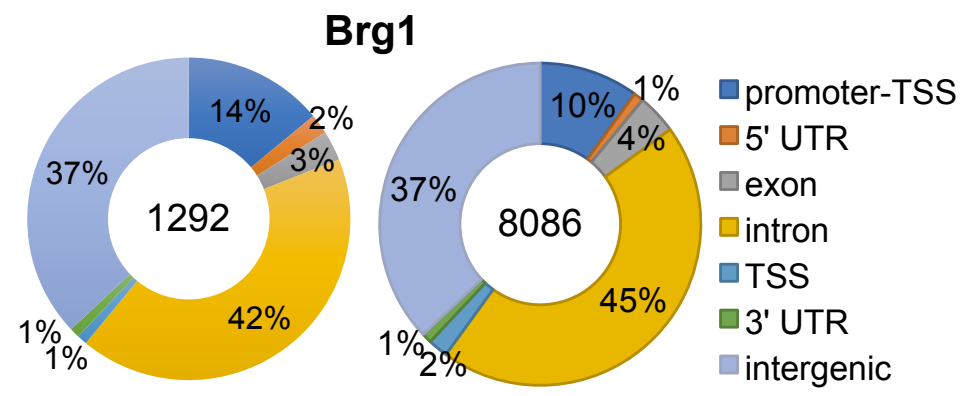

\section{Brg1/H3K27ac}

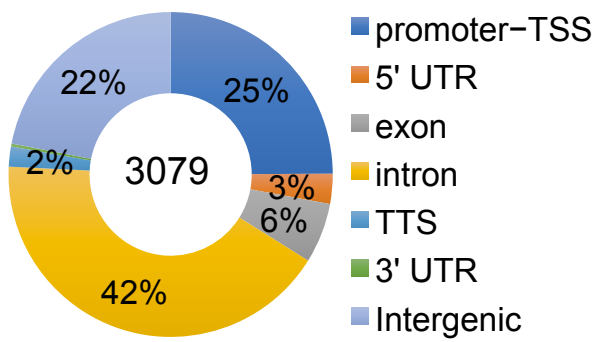

B

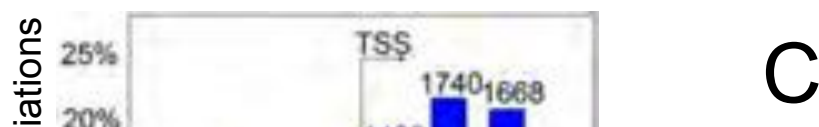

$\begin{array}{lccc} & \text { Motif } & \text { No. } & P \\ \text { CCGAGTTATT } & \text { bZIP } & 112 & 1 \mathrm{e}-14 \\ \text { CATACCCCCG } & \text { ZnF } & 230 & 1 \mathrm{e}-12 \\ \text { CGGTACAAA } & \text { sox4 } & 22 & 1 \mathrm{e}-12 \\ \text { CTITGTCGCG } & \text { sox10 } & 191 & 1 \mathrm{e}-10\end{array}$

Distance to TSS $(\mathrm{kb})$

D - $\log 10$ (Binomial $p$ value)

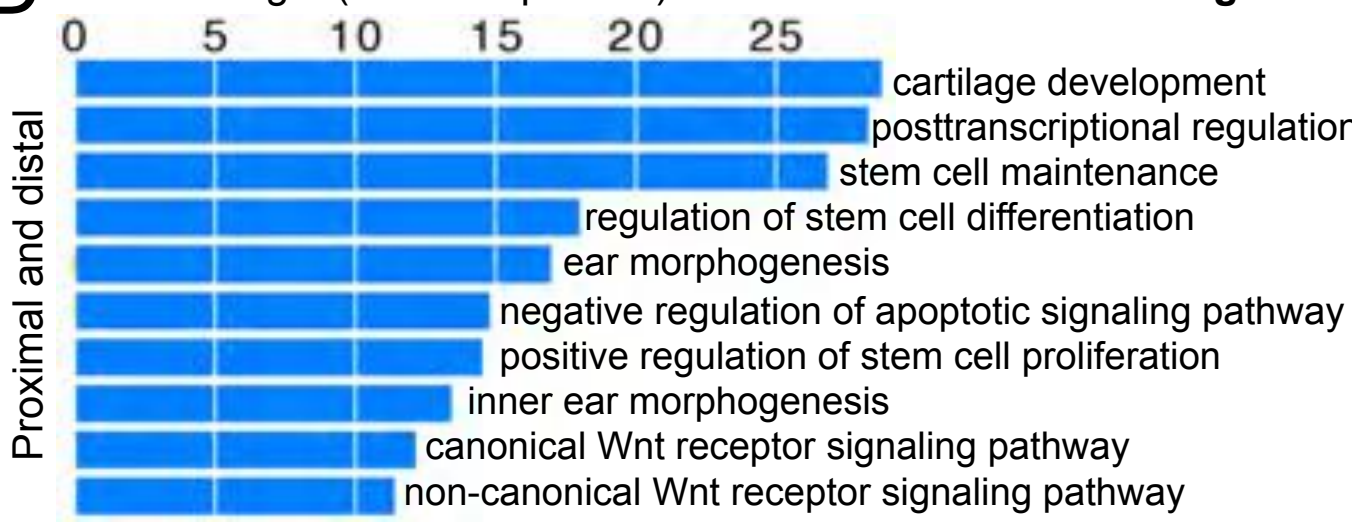

\section{GO Biological Process}

Binominal Fold Enrichment

GO Biological Process

\section{$\begin{array}{lllllllll}0 & 50 & 100 & 150 & 200 & 250 & 300 & 350 & 400\end{array}$}

\begin{tabular}{ll}
\hline & establishment of planar polarity
\end{tabular}

\section{cochlea morphogenesis}

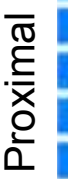
morphogenesis of a polarized epithelium

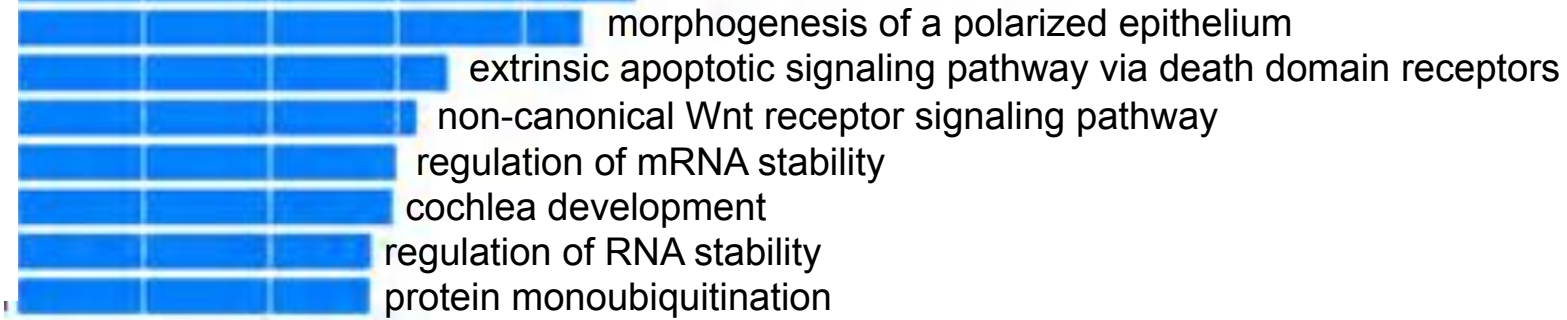

E Mycn

Cond1

(Chr7:144925-144945 kb)
Foxi1

(Chr11:34195-34215 kb)

(Chr7:12930-12950 kb)

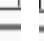

Brg1

H3K27ac

A...... Mtatithide.

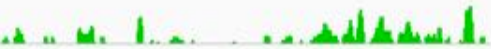

$\lg G$
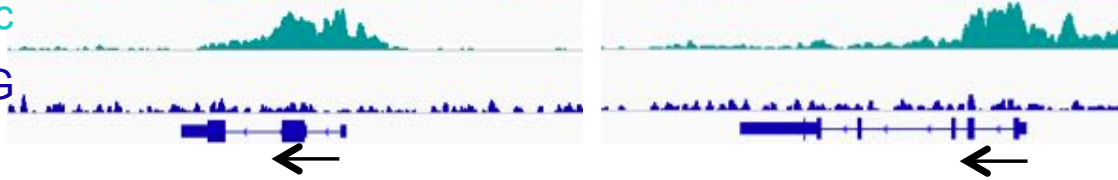
A Eya1

Chr1: 14,000 kb

$14,500 \mathrm{~kb}$

$15,000 \mathrm{~kb}$

Brg1

H3K27ac

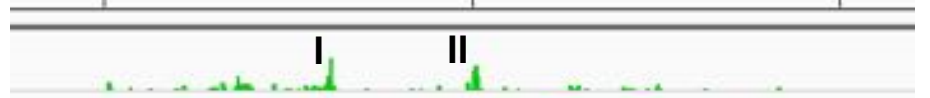

$\lg G$

Sox2
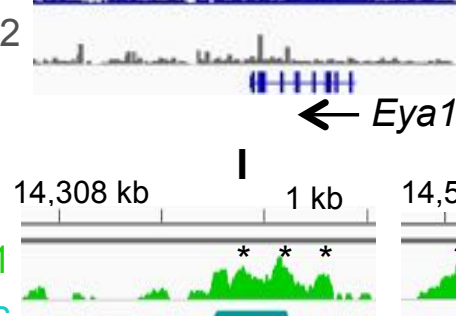

II

Trpa1

$\mathrm{Brg} 1$

H3K27ac

$\lg G$

Sox2

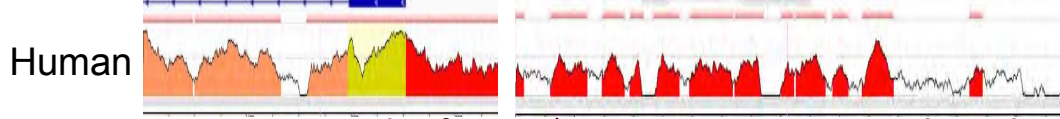

Primers:

B
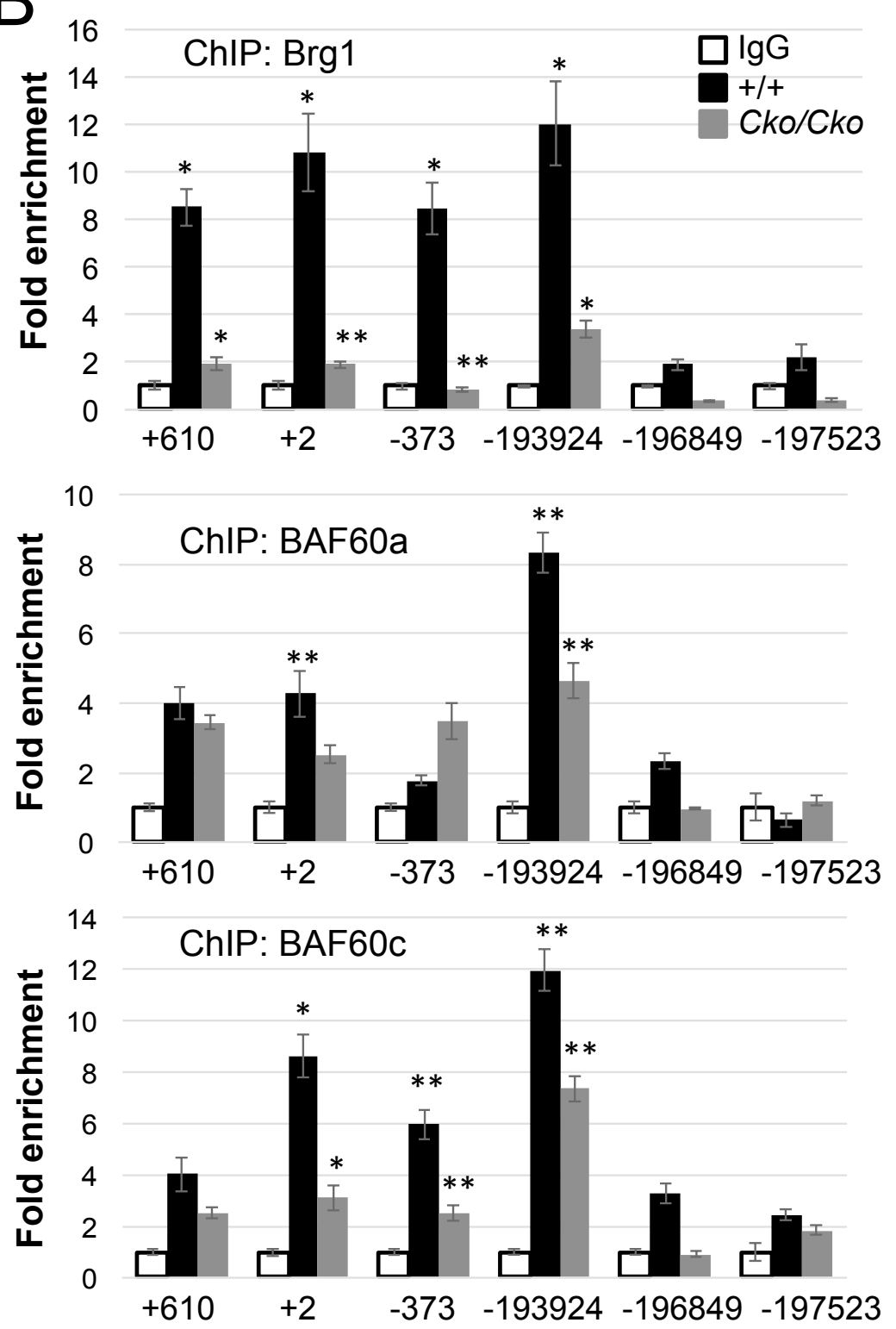

Cp27kip1

Chr6: $134,880 \mathrm{~kb}$

$134,940 \mathrm{~kb}$

Brg1

H3K27ac

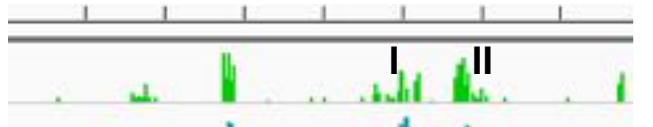

$\lg G$
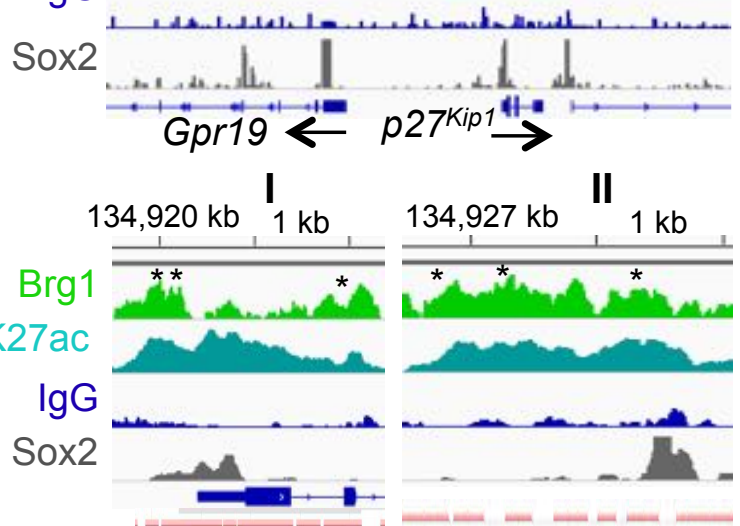

Human

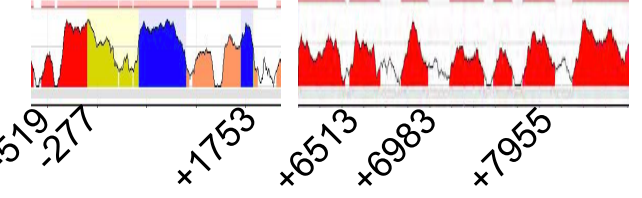

$D_{16}$

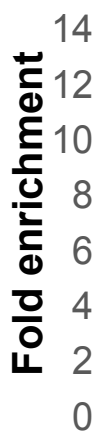

ChIP: Brg1
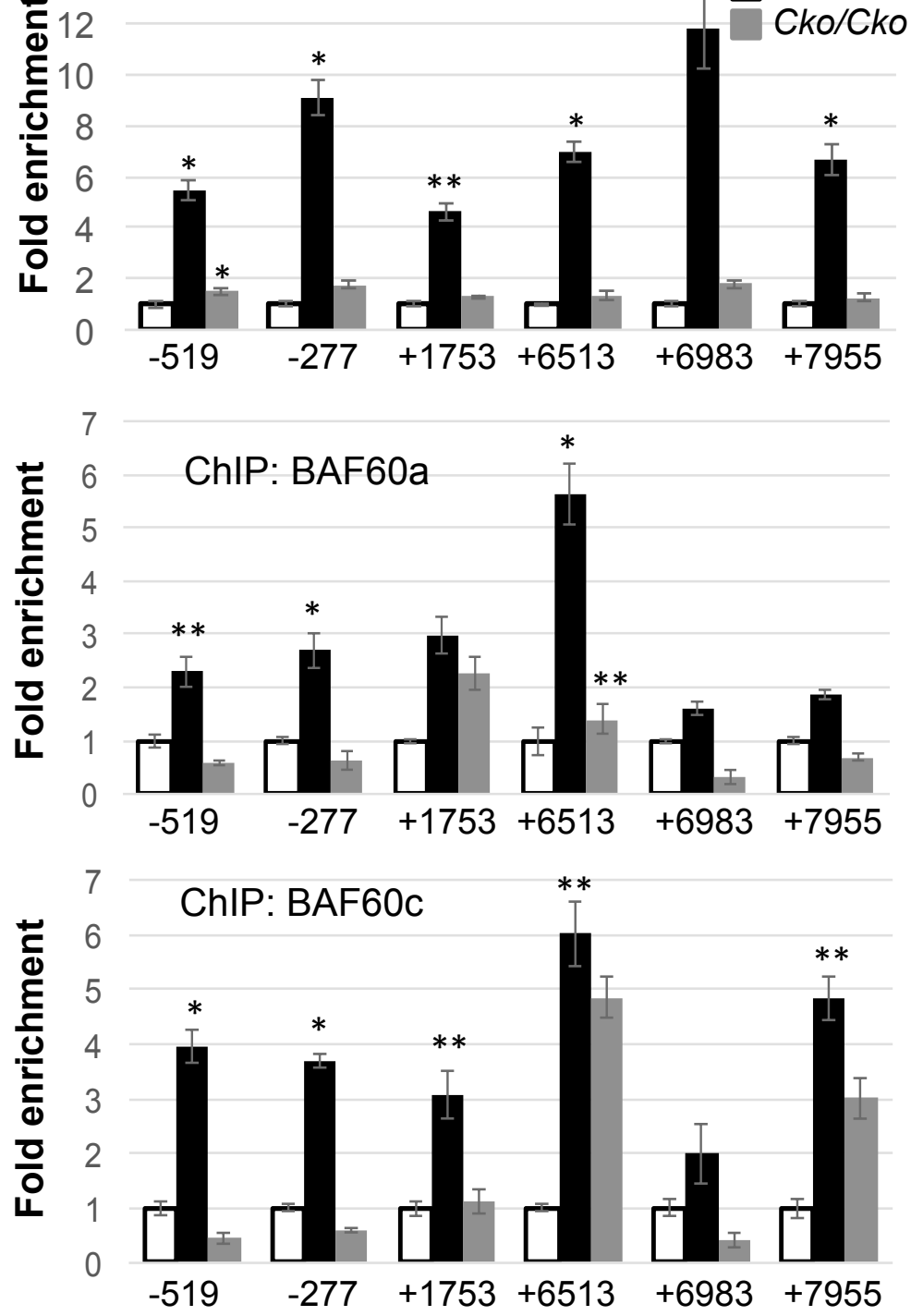


\section{Supplemental Figure legends}

Figure supplement 1. Immunostaining confirms selective deletion of Brg1 in the otocyst and cochlear epithelium using Eya1 ${ }^{\text {CreERT2 }}$. (A) Anti-Brg1 immunostaining on P0 cochlear section. SPG, spiral ganglion; IHC, inner hair cell; OHCs, outer hair cells. (B-G) Anti-Brg1 immunostaining on otic sections from Eyal ${ }^{\text {CreERT2 }}$ (B,D,F) and Eyal $^{\text {CreERT2 }}{ }^{\text {Brgl }} 1^{f l f l}$ littermate $(\mathrm{C}, \mathrm{E}, \mathrm{G})$ embryos at E10.5 (given tamoxifen at E8.5-9.5; B,E), E11.5 (given tamoxifen at E9.0-E10.0; C,F) and E14.5 (given tamoxifen at E11.5$12.5 ; \mathrm{D}, \mathrm{G})$ showing reduction of Brg1 in the ventral region of the mutant otocyst or in the primordial organ of Corti (bracket). As Eyal is also strongly expressed in surrounding periotic mesenchyme, this also led to removal of Brg1 in Eya1-expressing periotic mesenchyme. (C,E, compare with B,D). Scale bars: $50 \mu \mathrm{m}$.

Figure supplement 2. Conditional deletion of Sox2 in the otic placode does not alter the pattern of Dlx5, Eya1 and Six1 expression. (A,B) Immunostaining for Sox2 confirms deletion of Sox 2 in E10.5 otocyst using Eyal ${ }^{\text {CreERT2 (tamoxifen given from }}$ E7.5-8.5). (C,D) In situ hybridization on transverse sections of Eyal ${ }^{\text {CreERT2 }}$ and Sox $2^{C k o / C k o}$ otocysts for Dlx5 (C,D), Eyal (E,F) and Sixl (G,H). Abb.: a, anterior; d, dorsal; p, posterior; v, ventral. Scale bars: $50 \mu \mathrm{m}$.

Figure supplement 3. Brg1 is necessary for normal cell proliferation and survival in the cochlear sensory epithelium. (A) EdU-incorporation (red) in E13.5-14.0 wild-type and $\mathrm{Brg} 1^{\mathrm{Cko} / \mathrm{Cko}}$ cochlea given tamoxifen and EdU at E11.5. Sections were coimmunostained with Sox2 (green). (B) TUNEL analysis of cochlea of wild-type and 
$B r g 1^{C k o / C k o}$ embryos. Statistical analysis of EdU-labeled Sox $2^{+}$cells or apoptotic cells from 3 embryos per genotype showing the average number ( \pm standard deviations) of Sox $2^{+} / \mathrm{EdU}^{+}$cells or apoptotic cells per section $(6 \mu \mathrm{m})$ or per cochlea. $P=0.0028$ for B. $P$ value was established for $+/+$ and $B r g I^{C k o / C k o}$ using Two-tailed Student's $t$-test. Scale bars: $50 \mu \mathrm{m}$.

Figure supplement 4. Disruption of hair and supporting cell differentiation in Brgl ${ }^{\text {Cko/Cko }}$ cochlea. Immunostaining for Myo7a (green) and Prox 1 (red) on cochlear sections from E18.5 inner ears of wild-type and $\mathrm{Brgl}{ }^{\mathrm{Cko} / \mathrm{Cko}}$ littermates (given tamoxifen at E11.5-12.5. Scale bars: $30 \mu \mathrm{m}$.

Figure supplement 5. Brg1 physically interacts with Six1 and Sox2 and differential and overlapping patterns of BAF60a/c, BAF170 and BAF155 expression in the developing cochlea. (A) CoIP analysis. Nuclear extracts were prepared from E13.5 or E12.5 cochleae. Antibodies for IP and western blot are indicated. Input was $5 \%$ of the amount used for IP. Arrow indicates no interaction between BAF60b and Six1. (B,C) Immunostaining for BAF60a $(\mathrm{B})$ or BAF60c $(\mathrm{C})$ on cochlear sections of wild-type embryo at E18.5. (D,E) Immunostaining for BAF170 (D,F) or BAF155 (E,G) on cochlear sections of wild-type embryo at E14.5 or E18.5. Brackets mark the primordial organ of Corti. SPG, spiral ganglion. Scale bars: $30 \mu \mathrm{m}$ (B,C), $50 \mu \mathrm{m}$ (D-G).

Figure supplement 6. Genomic binding features of Brg1 and H3K27ac in E13.5 cochlea. (A) Genomic distribution of H3K27ac-enriched regions. Peaks were called with 
the MACS program. (B) Distribution of H3K27ac peaks relative to TSSs. (C) Differential enrichment for functional annotation terms associated Brg1-enriched regions. Shown are the top 5 enriched "molecular functions" associated with the 1292 or 8086 Brg1-enriched regions or specific to the $3079 \mathrm{Brg} 1$ regions with H3K27ac association. (D) Genomic view showing Brg1 enrichment at Myc, Cyclin D2 and Sixl/4 but not at Six6 locus.

\section{Figure supplement 7. Brg1 associates to both proximal and distal sites of key loci.} Genomic view showing Brg1 enrichment at genes involved in different pathways regulating sensory cell development. Wnt5a and Sfrp2 - involved in Wnt-signaling, Heyl and Lfng - involved in Notch signaling, Prox 1 - a marker specific for supporting cells; Atohl — critical for hair cell differentiation.

Figure supplement 8. Brg1-bound regions at the Six1, Sox2 and Eya4 loci and ChIP assays confirm Brg1 enrichment at these loci. (A-C) Genomic view showing Brg1bound regions at distal regions of the Six1, Sox2 and Eya4 loci. Regions selected for ChIP assays (corresponding to the $\mathrm{Brg} 1$ signals marked by asterisks) for each gene are indicated. At the Sox2 locus, four sites within an intergenic Brg1-bound region (located $\sim 691 \mathrm{~kb}$ downstream of Sox2; boxed by dashed line) were selected for ChIP assays. This region was also recovered in the Brg1 ChIP-seq on E11.5 forbrain (Attanasio et al., 2014) (red bar). Primers for amplifying each element are listed on Supplementary file 4. (D-F) ChIP-qPCR data using chromatin derived from otocysts of wild-type or $B r g 1^{C k o / C k o}$ littermates at E10.5 (given tamoxifen at E8.0-9.0) and anti-Brg1. Representative of three independent experiments, $\mathrm{n}=3$ for each group, ${ }^{*} P<0.01,{ }^{*} P<0.05$ determined by 
Student's t-test).

Attanasio, C., Nord, A.S., Zhu, Y., Blow, M.J., Biddie, S.C., Mendenhall, E.M., Dixon, J., Wright, C., Hosseini, R., Akiyama, J.A., et al. (2014). Tissue-specific SMARCA4 binding at active and repressed regulatory elements during embryogenesis. Genome Res 24, 920-929. 

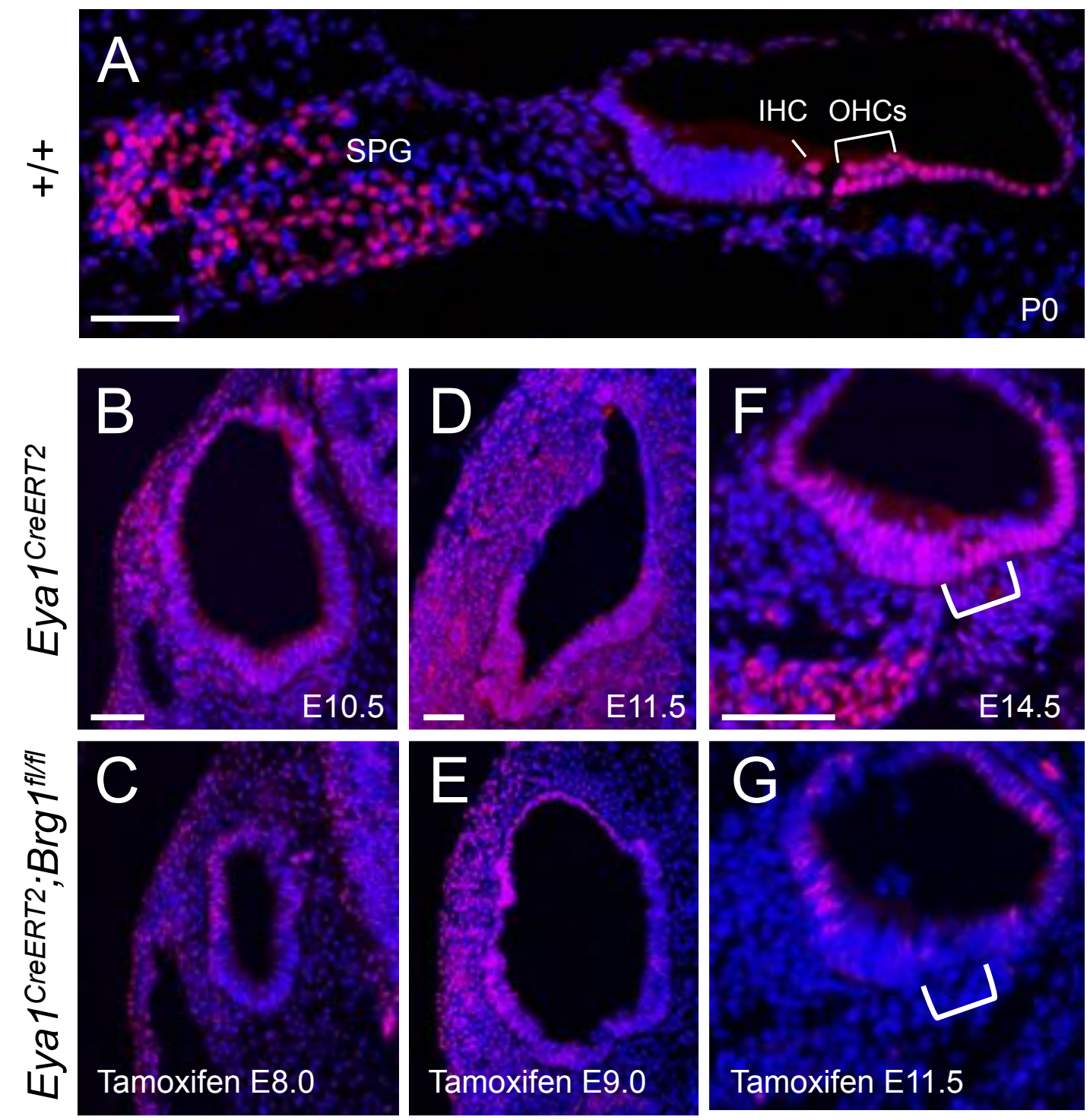
Eya1CreERT2
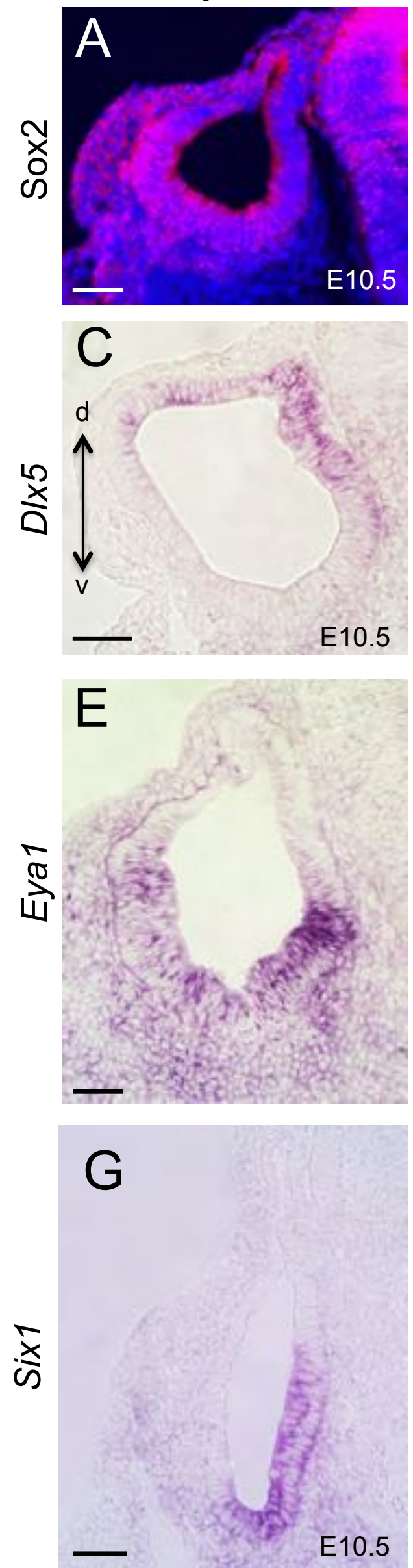

Eya1CreERT2;Sox ${ }^{\text {ff/fI }}$
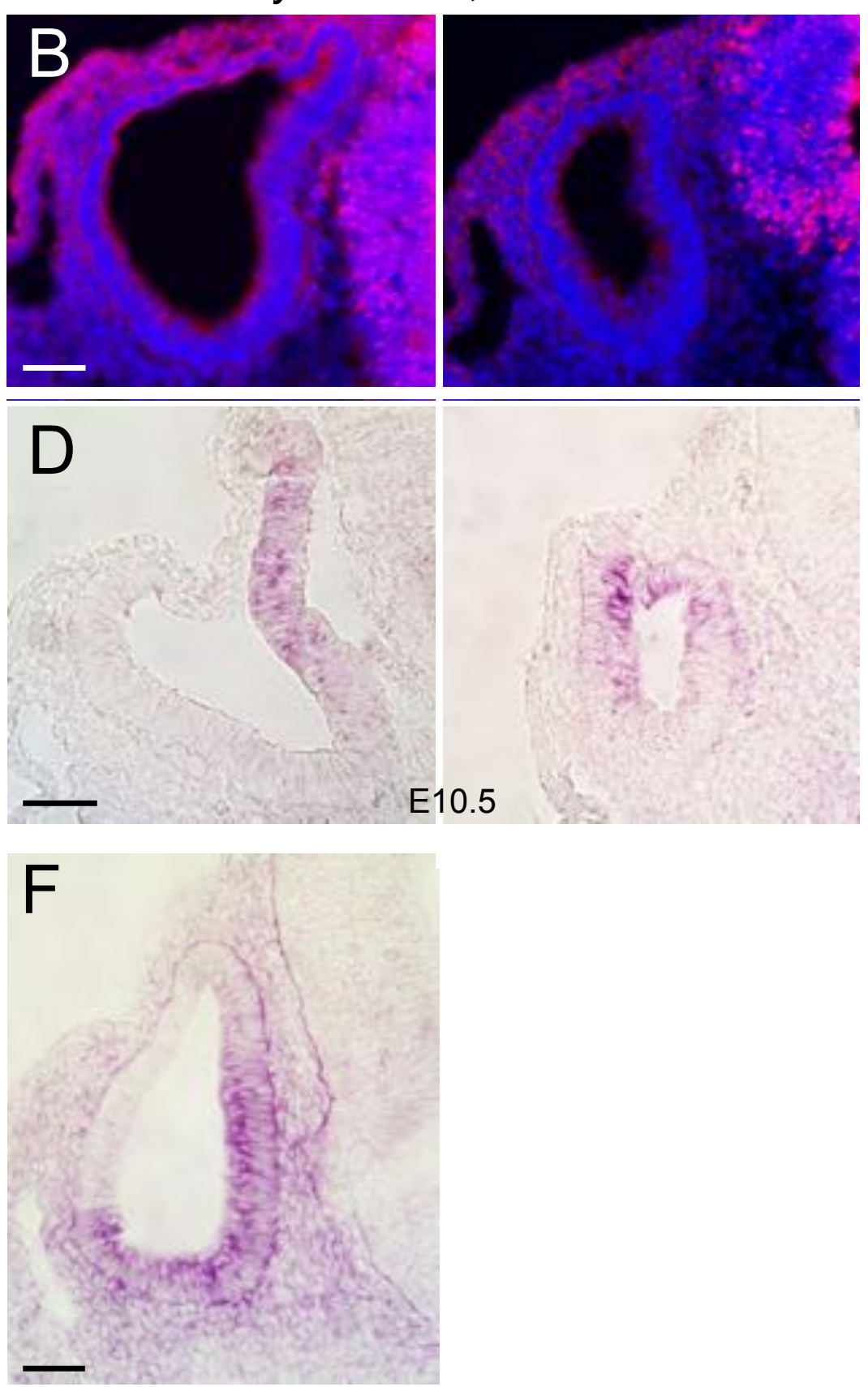

$\mathrm{H}$ 

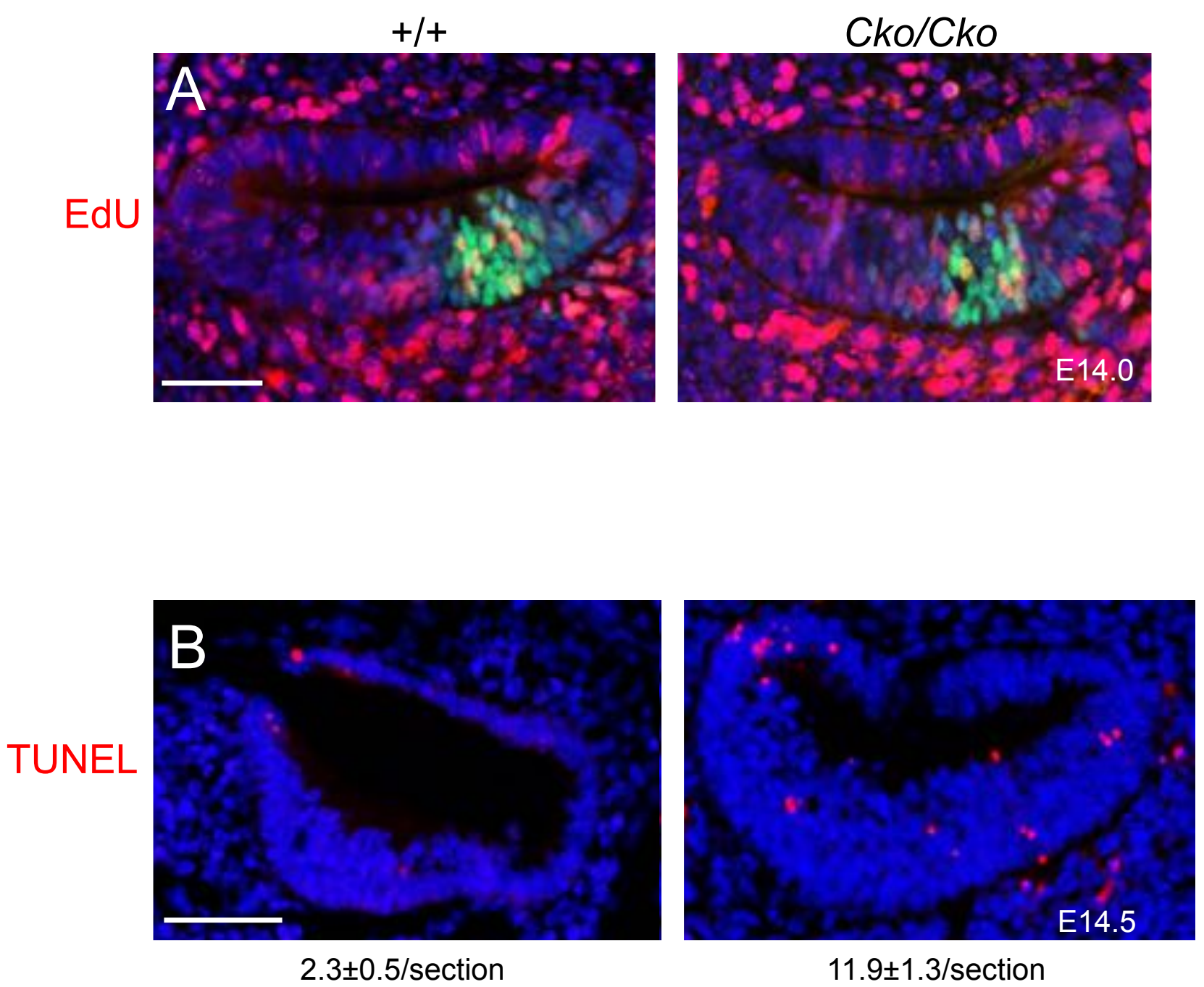


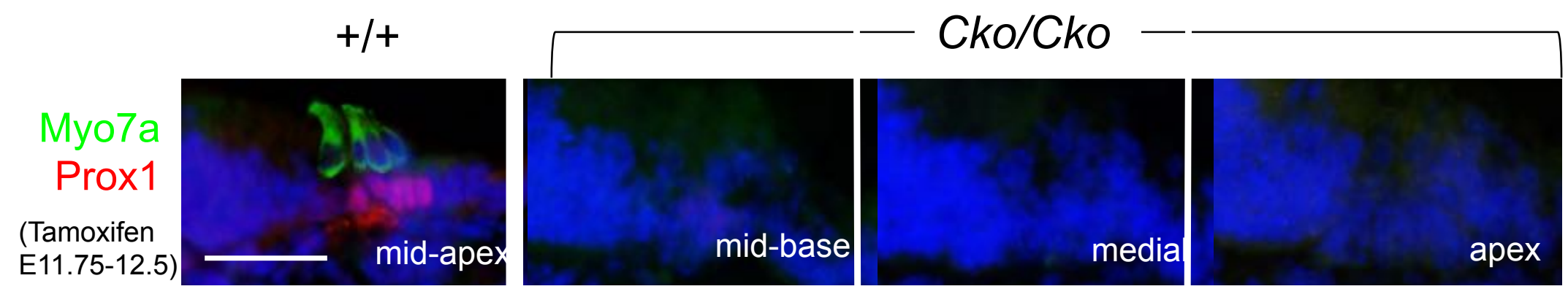




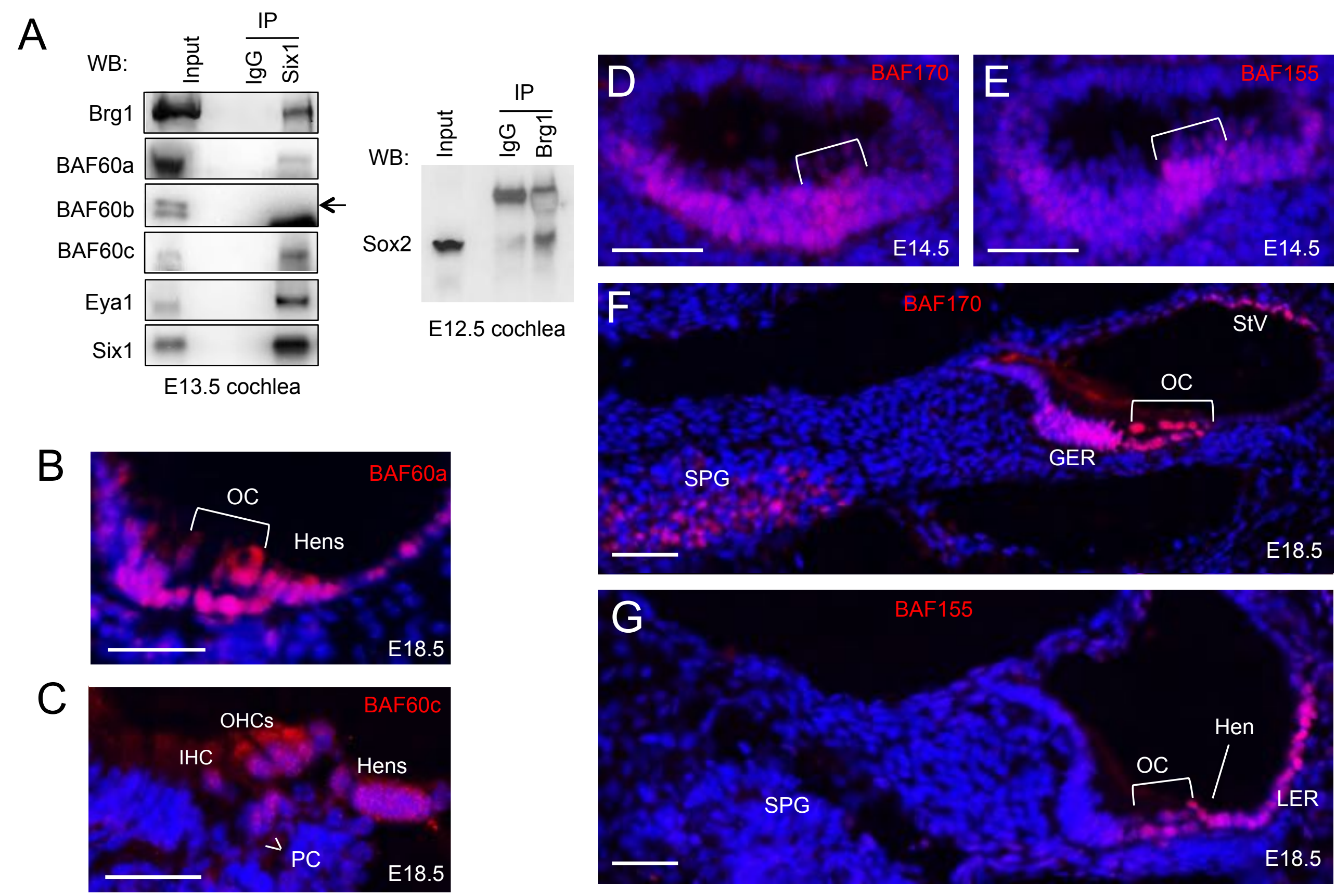


A
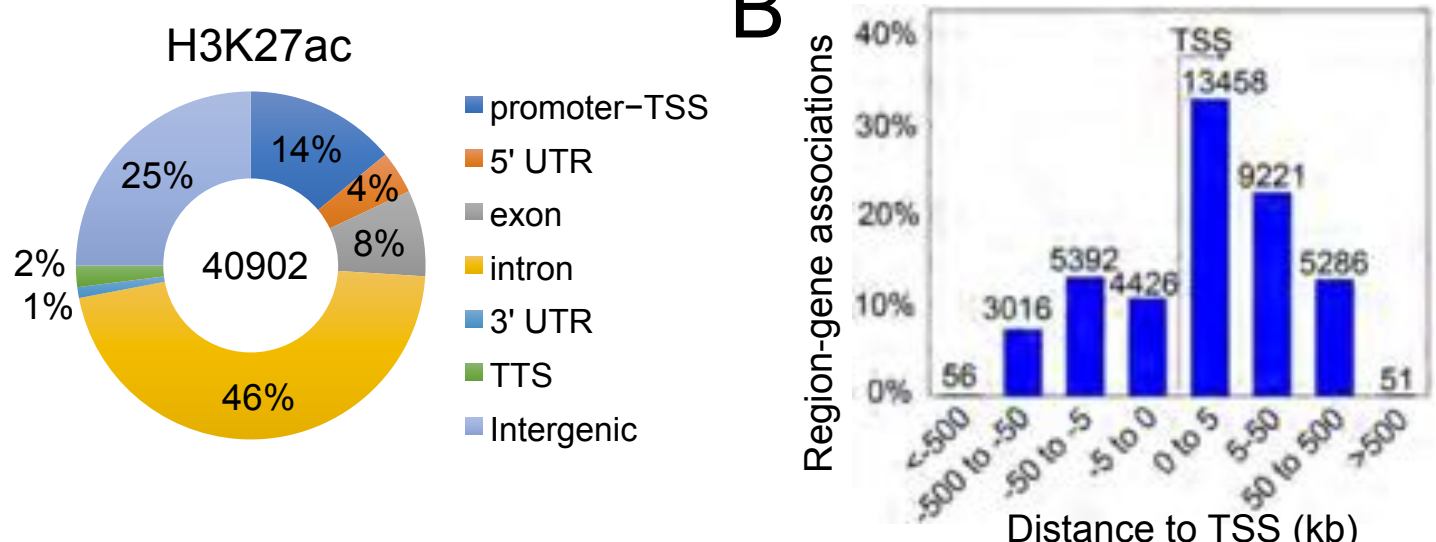

C Binomial Fold Enrichment

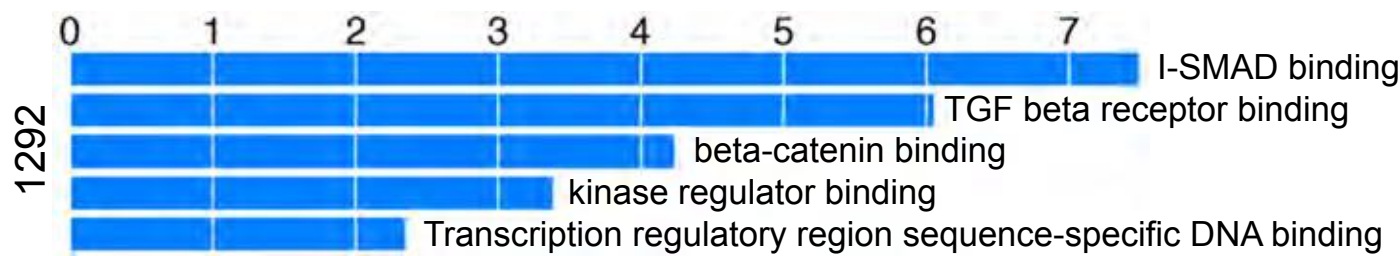

\section{GO Molecular Function}

Transcription regulatory region sequence-specific DNA binding

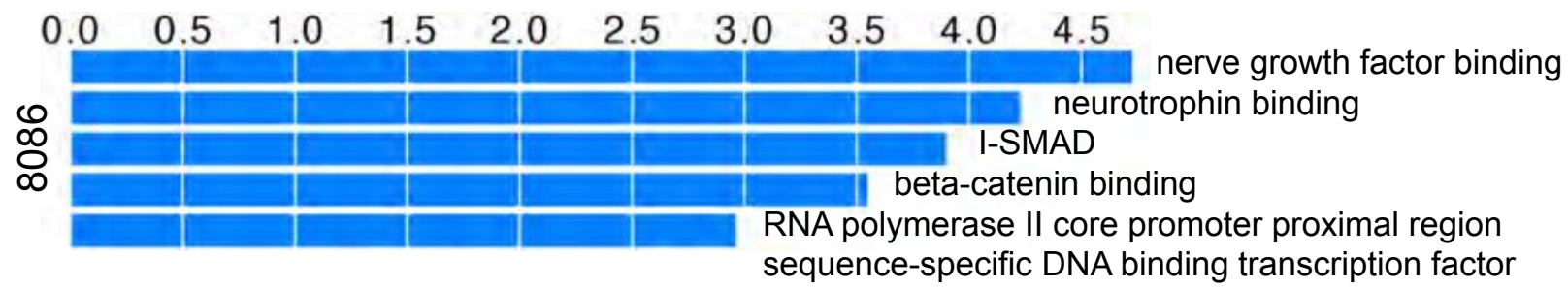

$-\log 10$ (Binomial $p$ value)

\section{GO Molecular Function}
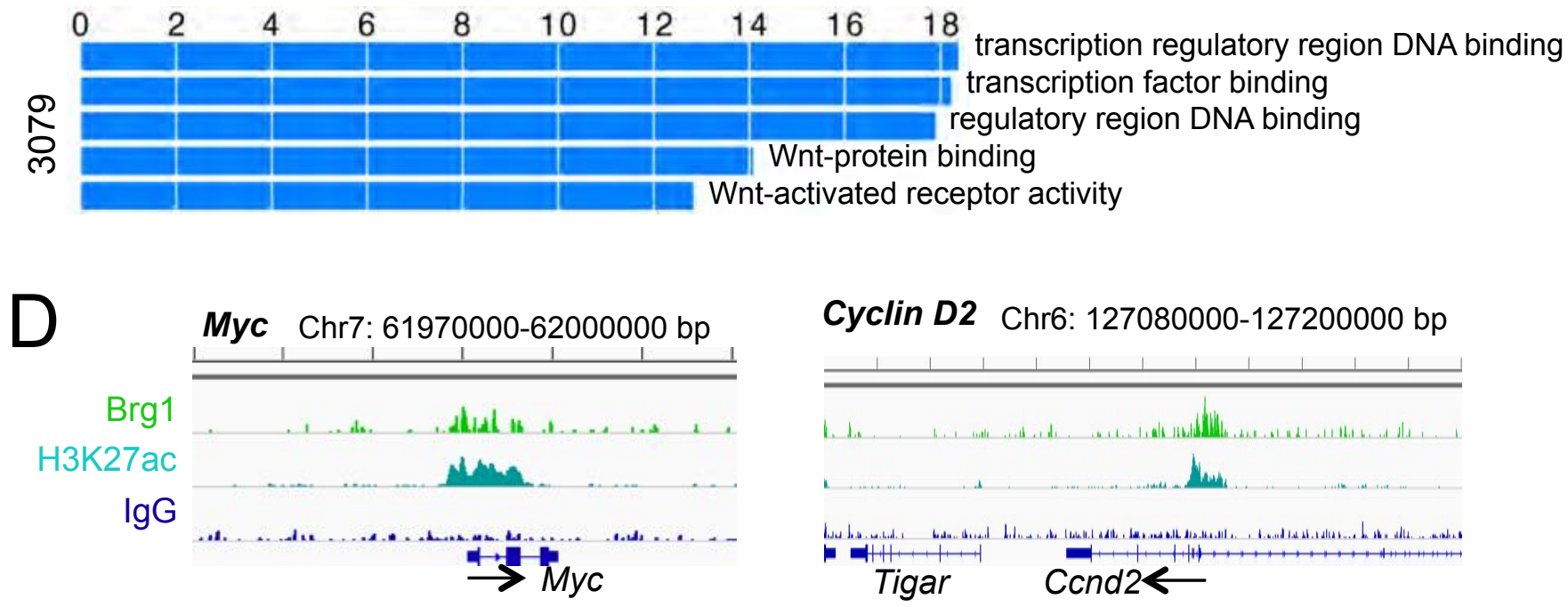

Cyclin D2 Chr6: 127080000-127200000 bp

Six1/4/6 Chr12: 72900000-73150000 bp

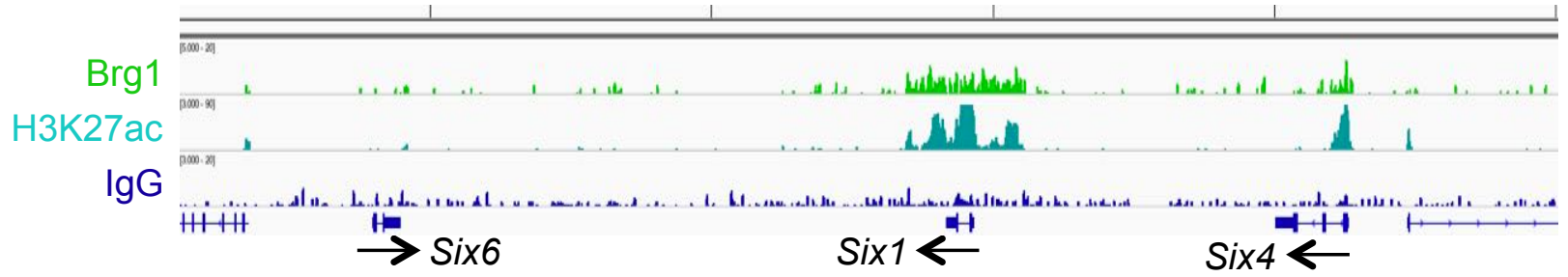


Wnt5a Chr14: 28470000-28580000 bp

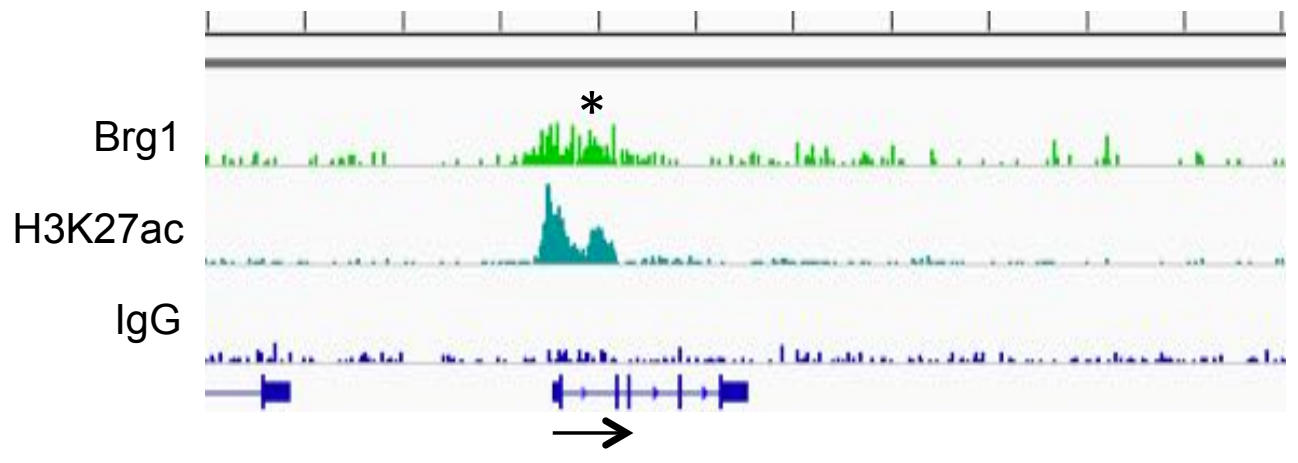

Hey1 Chr3: 8650000-8680000 bp

\begin{tabular}{lllll}
1 & 1 & 1 & 1 \\
\hline
\end{tabular}

Brg1

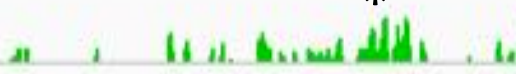

H3K27ac

$\lg G$

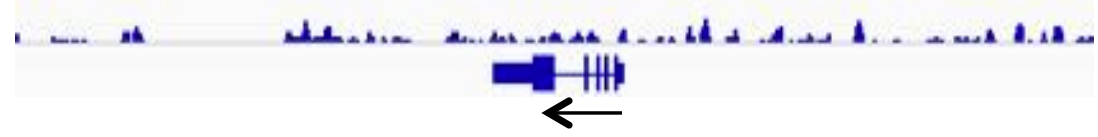

Prox1 Chr1: 190150000-190190000 bp

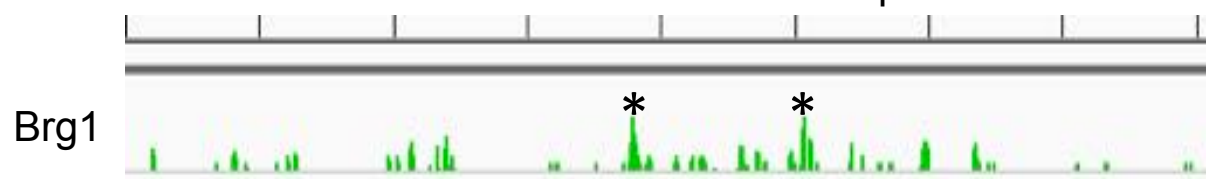

H3K27ac

$\lg G$

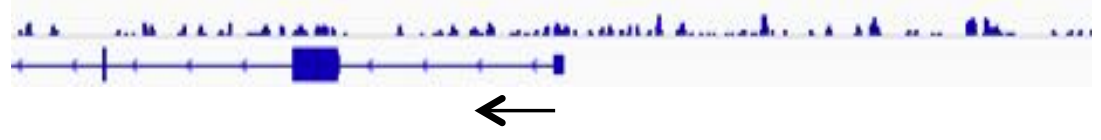

Sfrp2 Chr3: 83745000-83795000 bp

$*$

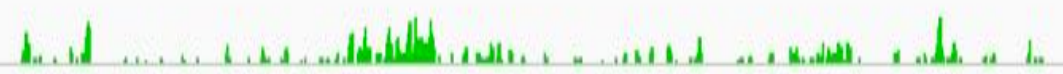

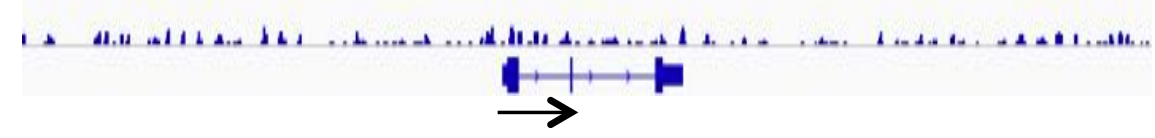

Lfng Chr5: 140595000-140625000 bp

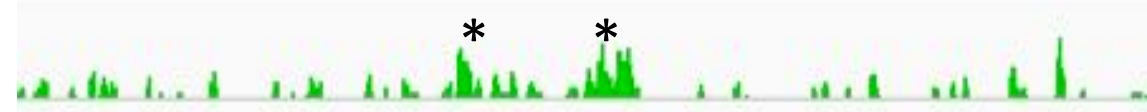

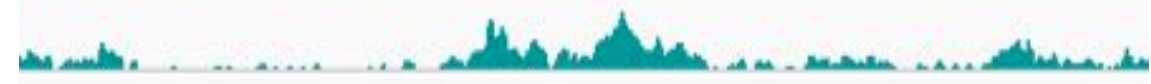

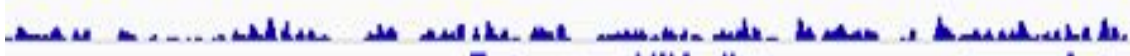

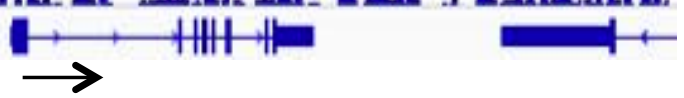

Atoh1 Chr6: 64725000-64737000 bp

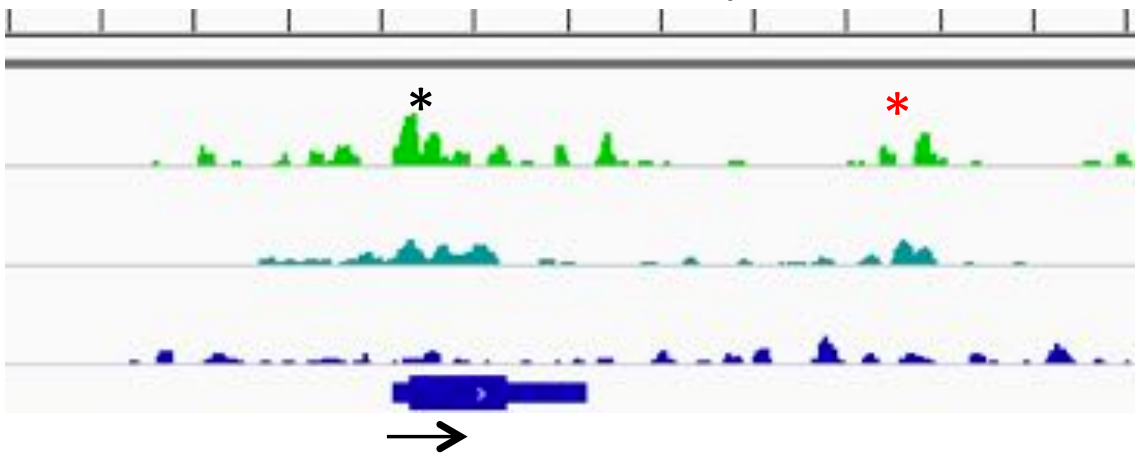


A

Six1 Chr12: 73025000-73065000 bp

$\mathrm{Brg} 1$

$\mathrm{H} 3 \mathrm{~K} 27 \mathrm{ac}$

$\lg G$

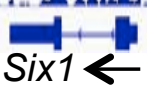

B

B Sox2 Chr3: $34000000-36000000 \mathrm{bp}$

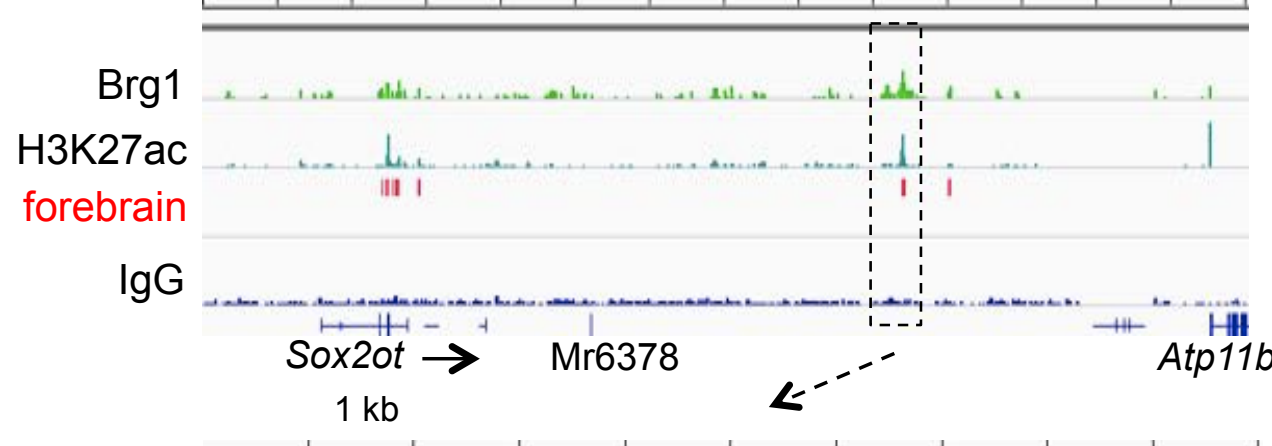

Brg1

H3K27ac

forebrain

C

$\lg G$

\section{Eya4 Chr10: $23330000-23370000$ bp}

$\mathrm{Brg} 1$

$* * *$

H3K27ac

$\lg G$

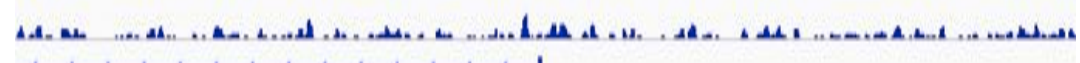

D

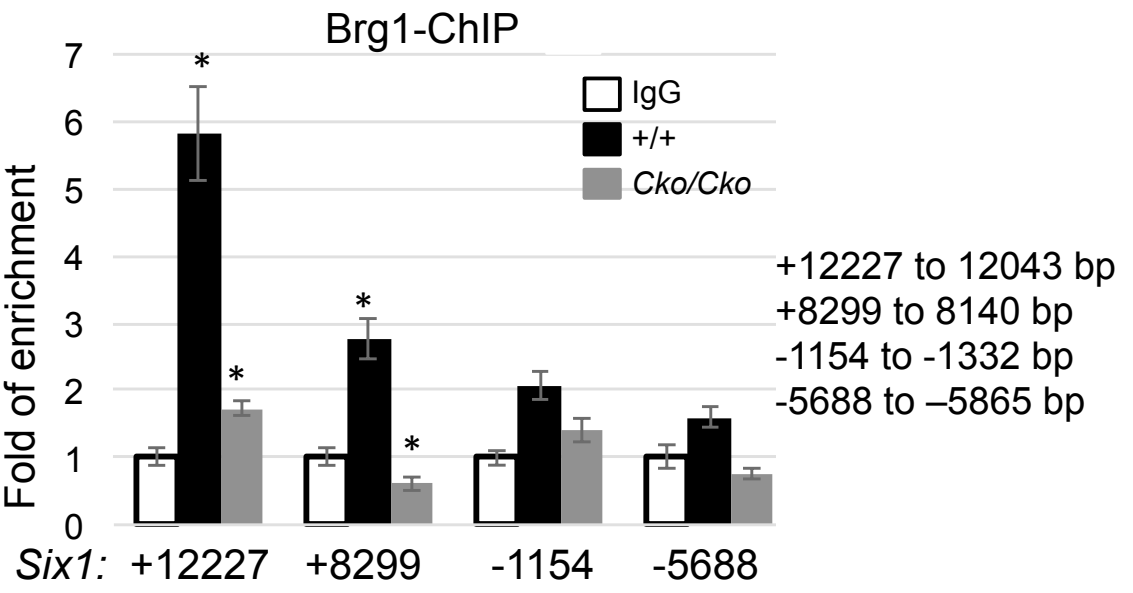

$E$
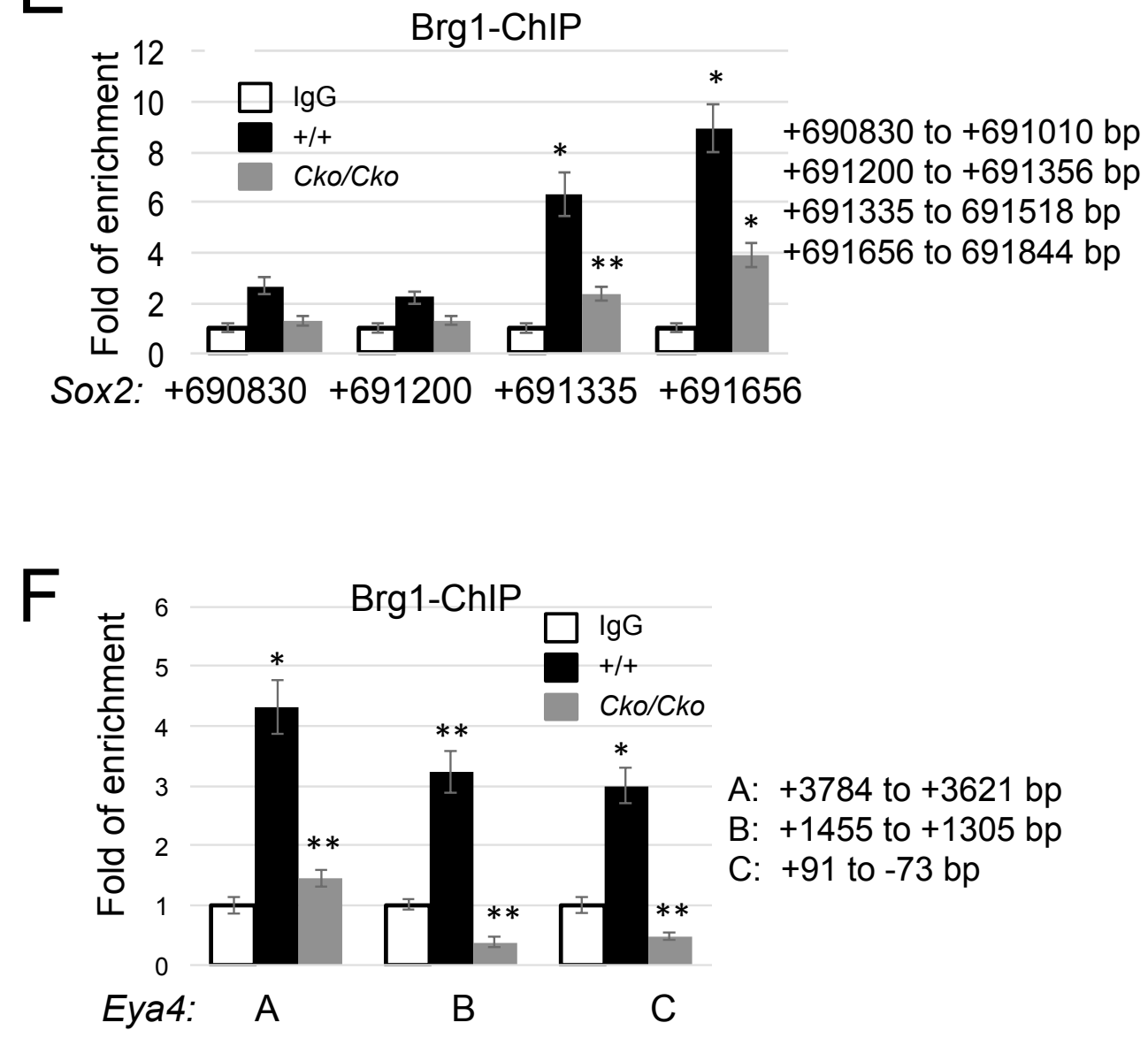